TAIWANESE JOURNAL OF MATHEMATICS

Vol. 12, No. 6, pp. 1401-1432, September 2008

This paper is available online at http://www.tjm.nsysu.edu.tw/

\title{
A NEW HYBRID-EXTRAGRADIENT METHOD FOR GENERALIZED MIXED EQUILIBRIUM PROBLEMS, FIXED POINT PROBLEMS AND VARIATIONAL INEQUALITY PROBLEMS
}

\author{
Jian-Wen Peng and Jen-Chih Yao* \\ Dedicated to Professor Wataru Takahashi on the occasion of his 65th birthday
}

\begin{abstract}
In this paper, we introduce a new iterative scheme based on the hybrid method and the extragradient method for finding a common element of the set of solutions of a generalized mixed equilibrium problem and the set of fixed points of a nonexpansive mapping and the set of the variational inequality for a monotone, Lipschitz-continuous mapping. We obtain a strong convergence theorem for the sequences generated by these processes in Hilbert spaces. Based on this result, we also get some new and interesting results. The results in this paper generalize, extend and unify some well-known strong convergence theorems in the literature.
\end{abstract}

\section{INTRODUCTION}

Let $H$ be a real Hilbert space with inner product $\langle.,$.$\rangle and induced norm \|$. and let $C$ be a nonempty closed convex subset of $H$. let $B: C \rightarrow H$ be a nonlinear mapping and let $\varphi: C \rightarrow R$ be a function and $F$ be a bifunction from $C \times C$ to $R$, where $R$ is the set of real numbers. Then, we consider the following generalized mixed equilibrium problem: Finding $x \in C$ such that

$$
F(x, y)+\varphi(y)-\varphi(x)+\langle B x, y-x\rangle \geq 0, \forall y \in C
$$

Received May 6, 2008

2000 Mathematics Subject Classification: 47H05, 47H10, $47 \mathrm{H} 17$.

Key words and phrases: Generalized mixed equilibrium problem, Extragradient method, Hybrid method, Nonexpansive mapping, Monotone mapping, Variational inequality, Strong convergence, Fixed point.

This research was supported by the National Natural Science Foundation of China ( Grant No. 10471159), the Science and Technology Research Project of Chinese Ministry of Education (Grant No.206123), the Education Committee project Research Foundation of Chongqing (Grant No. KJ070816).

*Corresponding author. 
The set of solutions of (1.1) is denoted by $\operatorname{GMEP}(F, \varphi, B)$.

If $B=0$, then the generalized mixed equilibrium problem (1.1) becomes the following mixed equilibrium problem :

Finding $x \in C$ such that $F(x, y)+\varphi(y)-\varphi(x) \geq 0, \forall y \in C$.

Problem (1.2) was studied by Ceng and Yao [1]. The set of solutions of (1.2) is denoted by $\operatorname{MEP}(F, \varphi)$.

If $\varphi=0$, then the generalized mixed equilibrium problem (1.1) becomes the following generalized equilibrium problem:

$$
\text { Finding } x \in C \text { such that } F(x, y)+\langle B x, y-x\rangle \geq 0, \forall y \in C \text {. }
$$

Problem (1.2) was studied by Takahashi and Takahashi [2]. The set of solutions of (1.3) is denoted by $G E P(F, B)$.

If $\varphi=0$ and $B=0$, then the generalized mixed equilibrium problem (1.1) becomes the following equilibrium problem:

$$
\text { Finding } x \in C \text { such that } F(x, y) \geq 0, \forall y \in C \text {. }
$$

The set of solutions of (1.4) is denoted by $E P(F)$.

If $F(x, y)=0$ for all $x, y \in C$, the generalized mixed equilibrium problem (1.1) becomes the following generalized variational inequality problem:

$$
\text { Finding } x \in C \text { such that } \varphi(y)-\varphi(x)+\langle B x, y-x\rangle \geq 0, \forall y \in C \text {. }
$$

The set of solutions of (1.5) is denoted by $G V I(C, B, \varphi)$.

If $\varphi=0$ and $F(x, y)=0$ for all $x, y \in C$, the generalized mixed equilibrium problem (1.1) becomes the following variational inequality problem:

$$
\text { Finding } x \in C \text { such that }\langle B x, y-x\rangle \geq 0, \forall y \in C \text {. }
$$

The set of solutions of (1.6) is denoted by $V I(C, B)$.

If $B=0$ and $F(x, y)=0$ for all $x, y \in C$, the generalized mixed equilibrium problem (1.1) becomes the following minimize problem:

$$
\text { Finding } x \in C \text { such that } \varphi(y)-\varphi(x) \geq 0, \forall y \in C \text {. }
$$

The set of solutions of (1.7) is denoted by $\operatorname{Argmin}(\varphi)$.

The problem (1.1) is very general in the sense that it includes, as special cases, optimization problems, variational inequalities, minimax problems, Nash equilibrium problem in noncooperative games and others; see for instance, [1-4].

Recall that a mapping $S$ of a closed convex subset $C$ of $H$ is nonexpansive [5] if there holds that

$$
\|S x-S y\| \leq\|x-y\| \text { for all } x, y \in C .
$$


We denote the set of fixed points of $S$ by Fix $(S)$. Ceng and Yao [1] introduced an iterative scheme for finding a common element of the set of solution of problem (1.2) and the set of common fixed points of a family of infinitely nonexpansive mappings in a Hilbert space and obtained a strong convergence theorem. Takahashi and Takahashi [2] introduced an iterative scheme for finding a common element of the set of solution of problem (1.3) and the set of fixed points of a nonexpansive mapping in a Hilbert space and proved a strong convergence theorem.

Some methods have been proposed to solve the problem (1.4); see, for instance, [3, 4, 6-9, 26 and the references therein]. Recently, Combettes and Hirstoaga [6] introduced an iterative scheme of finding the best approximation to the initial data when $\operatorname{EP}(F)$ is nonempty and proved a strong convergence theorem. Takahashi and Takahashi [7] introduced an iterative scheme by the viscosity approximation method for finding a common element of the set of solutions of problem (1.4) and the set of fixed points of a nonexpansive mapping in a Hilbert space proved a strong convergence theorem. Su, Shang and Qin [8] introduced the following iterative scheme by the viscosity approximation method for finding a common element of the set of solutions of problem (1.4) and the set of fixed points of a nonexpansive mapping and the set of solutions of the variational inequality problem for an $\alpha$ inverse strongly monotone mapping in a Hilbert space. Starting with an arbitrary $x_{1} \in H$, define sequences $\left\{x_{n}\right\}$ and $\left\{u_{n}\right\}$ by

$$
\left\{\begin{array}{l}
F\left(u_{n}, y\right)+\frac{1}{r_{n}}\left\langle y-u_{n}, u_{n}-x_{n}\right\rangle \geq 0, \quad \forall y \in C, \\
x_{n+1}=\alpha_{n} f\left(x_{n}\right)+\left(1-\alpha_{n}\right) S P_{C}\left(u_{n}-\lambda_{n} A u_{n}\right), \forall n \in N .
\end{array}\right.
$$

They proved that under certain appropriate conditions imposed on $\left\{\alpha_{n}\right\},\left\{r_{n}\right\}$ and $\left\{\lambda_{n}\right\}$, the sequences $\left\{x_{n}\right\}$ and $\left\{u_{n}\right\}$ generated by (1.8) converge strongly to $z \in$ $F i x(S) \cap E P(F) \cap V I(C, A)$, where $z=P_{F i x(S) \cap E P(F) \cap V I(C, A)} f(z)$. Tada and Takahashi [9] introduced the following iterative scheme by the hybrid method for finding a common element of the set of solutions of problem (1.4) and the set of fixed points of a nonexpansive mapping in a Hilbert space. Starting with an arbitrary $x_{1} \in H$, define sequences $\left\{x_{n}\right\}$ and $\left\{u_{n}\right\}$ by

$$
\left\{\begin{array}{l}
u_{n} \in C, F\left(u_{n}, y\right)+\frac{1}{r_{n}}\left\langle y-u_{n}, u_{n}-x_{n}\right\rangle \geq 0, \quad \forall y \in C, \\
w_{n}=\left(1-\alpha_{n}\right) x_{n}+\alpha_{n} S u_{n} \\
C_{n}=\left\{z \in H:\left\|w_{n}-z\right\| \leq\left\|x_{n}-z\right\|\right\} \\
Q_{n}=\left\{z \in H:\left\langle x_{n}-z, x-x_{n}\right\rangle \geq 0\right\} \\
x_{n+1}=P_{C_{n}} \cap Q_{n} x
\end{array}\right.
$$


They proved that under certain appropriate conditions imposed on $\left\{\alpha_{n}\right\}$ and $\left\{r_{n}\right\}$, the sequences $\left\{x_{n}\right\}$ and $\left\{u_{n}\right\}$ generated by (1.9) converge strongly to $P_{F i x(S) \cap E P(F)} x$. Generally speaking, the algorithm suggested by Tada and Takahashi is based on the well-known type of method, namely, on the so-called hybrid or "outer-approximation" for solving fixed point problem. The idea of "hybrid" or "outer-approximation" types of methods was originally introduced by Haugazeau in 1968 and was successfully generalized and extended in recent papers of Bauschke and Combettes [10], [11], Burachik, Lopes and Svaiter [12], Combettes [13], Nakajo and Takahashi [14], and Solodov and Svaiter [15], Kikkawa and Takahashi [16], Iiduka and Takahashi [17].

On the other hand, for solving the variational inequality problem in the finitedimensional Euclidean $R^{n}$, Korpelevich [18] introduced the following so-called extragradient method:

$$
\left\{\begin{array}{l}
x_{1}=x \in C \\
y_{n}=P_{C}\left(x_{n}-\lambda A x_{n}\right), \\
x_{n+1}=P_{C}\left(x_{n}-\lambda A y_{n}\right),
\end{array}\right.
$$

for every $n=0,1,2, \ldots$, where $\lambda \in\left(0, \frac{1}{k}\right)$. He showed that if $V I(C, A)$ is nonempty, then the sequences $\left\{x_{n}\right\}$ and $\left\{y_{n}\right\}$, generated by (1.10), converge to the same point $z \in V I(C, A)$. The idea of the extragradient iterative process introduced by Korpelevich was successfully generalized and extended not only in Euclidean but also in Hilbert and Banach spaces; see, e. g., the recent papers of He, Yang and Yuan [19], Gárciga Otero and Iuzem [20], Solodov and Svaiter [21], Solodov [22]. Moreover, Zeng and Yao [23] and Nadezhkina and Takahashi [24] introduced an iterative process based on the extragradient method for finding the common element of the set of fixed points of nonexpansive mappings and the set of solutions of variational inequality problem for a monotone, Lipschitz-continuous mapping. Yao and Yao [25] introduced an iterative process based on the extragradient method for finding the common element of the set of fixed points of nonexpansive mappings and the set of solutions of variational inequality problem for an $\alpha$-inverse strongly monotone mapping. Plubtieng and Punpaeng [26] introduced an iterative process based on the extragradient method for finding the common element of the set of fixed points of nonexpansive mappings, the set of an equilibrium problem and the set of solutions of variational inequality problem for $\alpha$-inverse strongly monotone mappings.

Very recently, by combine a hybrid method with an extragradient method, Nadezhkina and Takahashi [27] introduced an iterative process as follows: 


$$
\left\{\begin{array}{l}
x_{1}=x \in C \\
y_{n}=P_{C}\left(x_{n}-\lambda_{n} A x_{n}\right) \\
z_{n}=\beta_{n} x_{n}+\left(1-\beta_{n}\right) S P_{C}\left(x_{n}-\lambda_{n} A y_{n}\right) \\
C_{n}=\left\{z \in C:\left\|z_{n}-z\right\|^{2} \leq\left\|x_{n}-z\right\|^{2}\right\} \\
Q_{n}=\left\{z \in C:\left\langle x_{n}-z, x-x_{n}\right\rangle \geq 0\right\} \\
x_{n+1}=P_{C_{n}} \cap Q_{n} x
\end{array}\right.
$$

for every $n=1,2, \ldots$. They proved that under certain appropriate conditions imposed on $\left\{\beta_{n}\right\}$ and $\left\{\lambda_{n}\right\}$, the sequences $\left\{x_{n}\right\},\left\{y_{n}\right\}$ and $\left\{z_{n}\right\}$ generated by (1.11) converge strongly to $z \in F i x(S) \cap V I(C, A)$. Ceng, Hadjisavvas and Yao [28] introduced the following iterative process by combining hybrid -extragradient method for finding a common element of the set of fixed points of a nonexpansive mapping and the set of solutions of the variational inequality problem for a monotone, Lipschitz-continuous mapping.

$$
\left\{\begin{array}{l}
x_{1}=x \in C \\
y_{n}=\left(1-\gamma_{n}\right) x_{n}+\gamma_{n} P_{C}\left(x_{n}-\lambda_{n} A x_{n}\right) \\
z_{n}=\left(1-\alpha_{n}-\beta_{n}\right) x_{n}+\alpha_{n} y_{n}+\beta_{n} S P_{C}\left(x_{n}-\lambda_{n} A y_{n}\right) \\
C_{n}=\left\{z \in C:\left\|z_{n}-z\right\|^{2} \leq\left\|x_{n}-z\right\|^{2}+\left(3-3 \gamma_{n}+\alpha_{n}\right) b^{2}\left\|A x_{n}\right\|^{2}\right\} \\
Q_{n}=\left\{z \in C:\left\langle x_{n}-z, x-x_{n}\right\rangle \geq 0\right\} \\
x_{n+1}=P_{C_{n}} \cap Q_{n} x
\end{array}\right.
$$

for every $n=1,2, \ldots$. They proved that under certain appropriate conditions imposed on $\left\{\alpha_{n}\right\},\left\{\beta_{n}\right\},\left\{\gamma_{n}\right\}$ and $\left\{\lambda_{n}\right\}$, the sequences $\left\{x_{n}\right\},\left\{y_{n}\right\}$ and $\left\{z_{n}\right\}$ generated by (1.12) converge strongly to $z \in F i x(S) \cap V I(C, A)$. If $\gamma_{n}=1$ and $\alpha_{n}=0$ for every $n=1,2, \ldots$, then (1.12) becomes (1.11). Ceng, Hadjisavvas and Yao pointed up taking more general sequences $\left\{\alpha_{n}\right\},\left\{\beta_{n}\right\}$ and $\left\{\gamma_{n}\right\}$ might improve the rate of convergence to a solution.

In the present paper, by using the well-known KKM technique we derive an important lemma which is a foundation for studying the generalized mixed equilibrium problem. Then, we introduce a new iterative scheme based on the extragradient method and the hybrid method for finding a common element of the set of solutions of a generalized mixed equilibrium problem, the set of fixed points of a nonexpansive mapping and the set of the variational inequality for a monotone, Lipschitz-continuous mapping. We obtain a strong convergence theorem for the sequences generated by these processes. Based on this result, we also get some new and interesting results. The results in this paper generalize, extend and unify some well-known strong convergence theorems in the literature. 


\section{Preliminaries}

Let $H$ be a real Hilbert space with inner product $\langle\cdot, \cdot\rangle$ and norm $\|\cdot\|$. Let $C$ be a nonempty closed convex subset of $H$. Let symbols $\rightarrow$ and $\rightarrow$ denote strong and weak convergence, respectively. In a real Hilbert space $H$, it is well known that

$$
\|\lambda x+(1-\lambda) y\|^{2}=\lambda\|x\|^{2}+(1-\lambda)\|y\|^{2}-\lambda(1-\lambda)\|x-y\|^{2}
$$

for all $x, y \in H$ and $\lambda \in[0,1]$.

For any $x \in H$, there exists a unique nearest point in $C$, denoted by $P_{C}(x)$, such that $\left\|x-P_{C}(x)\right\| \leq\|x-y\|$ for all $y \in C$. The mapping $P_{C}$ is called the metric projection of $H$ onto $C$. We know that $P_{C}$ is a nonexpansive mapping from $H$ onto $C$. It is also known that $P_{C} x \in C$ and

$$
\left\langle x-P_{C}(x), P_{C}(x)-y\right\rangle \geq 0
$$

for all $x \in H$ and $y \in C$.

It is easy to see that (2.1) is equivalent to

$$
\|x-y\|^{2} \geq\left\|x-P_{C}(x)\right\|^{2}+\left\|y-P_{C}(x)\right\|^{2}
$$

for all $x \in H$ and $y \in C$.

A mapping $A$ of $C$ into $H$ is called monotone if

$$
\langle A x-A y, x-y\rangle \geq 0
$$

for all $x, y \in C$. A mapping $A$ of $C$ into $H$ is called $\alpha$-inverse-strongly monotone if there exists a positive real number $\alpha$ such that

$$
\langle x-y, A x-A y\rangle \geq \alpha\|A x-A y\|^{2}
$$

for all $x, y \in C$. A mapping $A: C \rightarrow H$ is called $k$-Lipschitz-continuous if there exists a positive real number $k$ such that

$$
\|A x-A y\| \leq k\|x-y\|
$$

for all $x, y \in C$. It is easy to see that if $A$ is an $\alpha$-inverse-strongly-monotone mapping, then $A$ is monotone and Lipschitz-continuous. The converse is not true in general. The class of $\alpha$-inverse-strongly-monotone mappings does not contain some important classes of mappings even in a finite-dimensional case. For example, if the matrix in the corresponding linear complementarity problem is positively semidefinite, but not positively definite, then the mapping $A$ will be monotone and Lipschitz-continuous, but not $\alpha$-inverse-strongly-monotone (see [27]).

Let $A$ be a monotone mapping of $C$ into $H$. In the context of the variational inequality problem the characterization of projection (2.1) implies the following:

$$
u \in V I(C, A) \Rightarrow u=P_{C}(u-\lambda A u), \lambda>0,
$$


and

$$
u=P_{C}(u-\lambda A u) \text { for some } \lambda>0 \Rightarrow u \in V I(C, A) .
$$

It is also known that $H$ satisfies the Opial's condition [29], i.e., for any sequence $\left\{x_{n}\right\} \subset H$ with $x_{n} \rightarrow x$, the inequality

$$
\liminf _{n \rightarrow \infty}\left\|x_{n}-x\right\|<\liminf _{n \rightarrow \infty}\left\|x_{n}-y\right\|
$$

holds for every $y \in H$ with $x \neq y$.

A set-valued mapping $T: H \rightarrow 2^{H}$ is called monotone if for all $x, y \in H$, $f \in T x$ and $g \in T y$ imply $\langle x-y, f-g\rangle \geq 0$. A monotone mapping $T: H \rightarrow 2^{H}$ is maximal if its graph $G(T)$ of $T$ is not properly contained in the graph of any other monotone mapping. It is known that a monotone mapping $T$ is maximal if and only if for $(x, f) \in H \times H,\langle x-y, f-g\rangle \geq 0$ for every $(y, g) \in G(T)$ implies $f \in T x$. Let A be a monotone, $k$-Lipschitz-continuous mapping of $C$ into $H$ and let $N_{C} v$ be normal cone to $C$ at $v \in C$, i.e, $N_{C} v=\{w \in H:\langle v-u, w\rangle \geq 0, \forall u \in C\}$. Define

$$
T v= \begin{cases}A v+N_{C} v & \text { if } v \in C, \\ \emptyset & \text { if } v \notin C .\end{cases}
$$

Then $T$ is maximal monotone and $0 \in T v$ if and only if $v \in V I(C, A)$ (see [30]).

For each $B \subseteq H$, we denote by $\operatorname{conv}(B)$ the convex hull of $B$. A multivalued mapping $G: B \rightarrow 2^{H}$ is said to be a KKM map if, for every finite subset $\left\{x_{1}, x_{2}, \ldots, x_{n}\right\} \subseteq B$,

$$
\operatorname{conv}\left(x_{1}, x_{2}, \ldots, x_{n}\right) \subseteq \bigcup_{n=1}^{\infty} G\left(x_{i}\right) .
$$

We shall use the following results in the sequel.

Lemma 2.1. ([31]). Let $B$ be a nonempty subset of a Hausdorff topological vector space $X$ and let $G: B \rightarrow 2^{X}$ be a KKM map. If $G(x)$ is closed for all $x \in B$ and is compact for at least one $x \in B$, then $\bigcap_{x \in B} G(x) \neq \emptyset$.

Lemma 2.2. (see Proposition 5.3 in [32]). Let $C$ be a nonempty closed convex subset of a strictly convex Banach space $X$ and $S: C \rightarrow C$ a nonexpansive mapping with Fix $(S) \neq \emptyset$. Then Fix $(S)$ is closed and convex.

For solving the generalized mixed equilibrium problem and the mixed equilibrium problem, let us give the following assumptions for the bifunction $F, \varphi$ and the set $C$ :

(A1) $F(x, x)=0$ for all $x \in C$;

(A2) $F$ is monotone, i.e. $F(x, y)+F(y, x) \leq 0$ for any $x, y \in C$;

(A3) for each $y \in C, x \mapsto F(x, y)$ is weakly upper semicontinuous;

(A4) for each $x \in C, y \mapsto F(x, y)$ is convex; 
(A5) for each $x \in C, y \mapsto F(x, y)$ is lower semicontinuous;

(B1) For each $x \in H$ and $r>0$, there exist a bounded subset $D_{x} \subseteq C$ and $y_{x} \in C$ such that for any $z \in C \backslash D_{x}$,

$$
F\left(z, y_{x}\right)+\varphi\left(y_{x}\right)-\varphi(z)+\frac{1}{r}\left\langle y_{x}-z, z-x\right\rangle<0 ;
$$

(B2) $C$ is a bounded set;

(B3) For each $x \in H$ and $r>0$, there exist a bounded subset $D_{x} \subseteq C$ and $y_{x} \in C$ such that for any $z \in C \backslash D_{x}$,

$$
\varphi\left(y_{x}\right)-\varphi(z)+\frac{1}{r}\left\langle y_{x}-z, z-x\right\rangle<0 ;
$$

(B4) For each $x \in H$ and $r>0$, there exist a bounded subset $D_{x} \subseteq C$ and $y_{x} \in C$ such that for any $z \in C \backslash D_{x}$,

$$
F\left(z, y_{x}\right)+\frac{1}{r}\left\langle y_{x}-z, z-x\right\rangle<0 \text {. }
$$

Lemma 2.3. Let $C$ be a nonempty closed convex subset of $H$. Let $F$ be a bifunction from $C \times C$ to $R$ satisfying $(A 1)-(A 4)$ and let $\varphi: C \rightarrow R$ be a proper lower semicontinuous and convex function. For $r>0$ and $x \in H$, define $a$ mapping $T_{r}: H \rightarrow C$ as follows.

$$
T_{r}(x)=\left\{z \in C: F(z, y)+\varphi(y)-\varphi(z)+\frac{1}{r}\langle y-z, z-x\rangle \geq 0, \forall y \in C\right\}
$$

for all $x \in H$. Assume that either (B1) or (B2) holds. Then, the following results hold:

(1) For each $x \in H, T_{r}(x) \neq \emptyset$;

(2) $T_{r}$ is single-valued;

(3) $T_{r}$ is firmly nonexpansive, i.e, for any $x, y \in H$,

$$
\left\|T_{r}(x)-T_{r}(y)\right\|^{2} \leq\left\langle T_{r}(x)-T_{r}(y), x-y\right\rangle ;
$$

(4) $\operatorname{Fix}\left(T_{r}\right)=\operatorname{MEF}(F, \varphi)$;

(5) $\operatorname{MEF}(F, \varphi)$ is closed and convex.

Proof. Let $x_{0}$ be any given point in $H$. For each $y \in C$, we define

$$
G(y)=\left\{z \in C: F(z, y)+\varphi(y)-\varphi(z)+\frac{1}{r}\left\langle y-z, z-x_{0}\right\rangle \geq 0\right\} .
$$

Note that for each $y \in C, G(y)$ is nonempty since $y \in G(y)$. We shall prove that $G$ is a KKM map. Suppose that there exist a finite subset $\left\{y_{1}, y_{2}, \ldots, y_{n}\right\}$ of $C$ and 
$\mu_{i} \geq 0$ for all $i=1,2, \ldots, n$ with $\sum_{i=1}^{n} \mu_{i}=1$ such that $\hat{z}=\sum_{i=1}^{n} \mu_{i} y_{i} \notin G\left(y_{i}\right)$ for each $i=1,2, \ldots, n$. Then we have

$$
F\left(\hat{z}, y_{i}\right)+\varphi\left(y_{i}\right)-\varphi(\hat{z})+\frac{1}{r}\left\langle y_{i}-\hat{z}, \hat{z}-x_{0}\right\rangle<0
$$

for each $i=1,2, \ldots, n$. By (A4) and the convexity of $\varphi$, we have

$$
\begin{gathered}
0=F(\hat{z}, \hat{z})+\varphi(\hat{z})-\varphi(\hat{z})+\frac{1}{r}\left\langle\hat{z}-\hat{z}, \hat{z}-x_{0}\right\rangle \\
\leq \sum_{i=1}^{n} \mu_{i}\left[F\left(\hat{z}, y_{i}\right)+\varphi\left(y_{i}\right)-\varphi(\hat{z})\right]+\frac{1}{r}\left[\sum_{i=1}^{n} \mu_{i}\left\langle y_{i}-\hat{z}, \hat{z}-x_{0}\right\rangle\right]<0
\end{gathered}
$$

which is a contradiction. Hence, $G$ is a KKM map. Note that $\overline{G(y)}^{w}$ (the weak closure of $G(y)$ ) is a weakly closed subset of $C$ for each $y \in C$. Moreover, if (B2) holds, then $\overline{G(y)}^{w}$ is also weakly compact for each $y \in C$. If (B1) holds, then for $x_{0} \in H$, there exist a bounded subset $D_{x_{0}} \subseteq C$ and $y_{x_{0}} \in C$ such that for any $y \in C \backslash D_{x_{0}}$,

$$
F\left(z, y_{x_{0}}\right)+\varphi\left(y_{x_{0}}\right)-\varphi(z)+\frac{1}{r}\left\langle y_{x_{0}}-z, z-x_{0}\right\rangle<0 .
$$

This shows that

$G\left(y_{x_{0}}\right)=\left\{z \in C: F\left(z, y_{x_{0}}\right)+\varphi\left(y_{x_{0}}\right)-\varphi(z)+\frac{1}{r}\left\langle y_{x_{0}}-z, z-x_{0}\right\rangle \geq 0\right\} \subseteq D_{x_{0}}$.

And hence $\overline{G\left(y_{x_{0}}\right)}$ is weakly compact. Thus, in both cases, we can use Lemma 2.1 and have $\cap_{y \in C} \overline{G(y)}^{w} \neq \emptyset$.

Next we shall prove that $\overline{G(y)}^{w}=G(y)$ for each $y \in C$; i.e., $G(y)$ is weakly closed. Let $z \in \overline{G(y)}^{w}$ and $z_{m}$ be a sequence in $G(y)$ such that $z_{m} \rightarrow z$. Then,

$$
F\left(z_{m}, y\right)+\varphi(y)-\varphi\left(z_{m}\right)+\frac{1}{r}\left\langle y-z_{m}, z_{m}-x_{0}\right\rangle \geq 0
$$

Since $\|\cdot\|^{2}$ is weakly lower semicontinuous, we have

$$
\begin{aligned}
& \limsup _{m \rightarrow \infty}\left\langle y-z_{m}, z_{m}-x_{0}\right\rangle \\
= & \limsup _{m \rightarrow \infty}\left[\left\langle y-z_{m},-x_{0}\right\rangle+\left\langle y, z_{m}\right\rangle-\left\|z_{m}\right\|^{2}\right] \\
= & \lim _{m \rightarrow \infty}\left\langle z_{m}-y, x_{0}\right\rangle+\lim _{m \rightarrow \infty}\left\langle y, z_{m}\right\rangle-\lim i n f_{m \rightarrow \infty}\left\|z_{m}\right\|^{2} \\
\leq & \left\langle z-y, x_{0}\right\rangle+\langle y, z\rangle-\|z\|^{2} \\
= & \left\langle z-y, x_{0}-z\right\rangle .
\end{aligned}
$$


It follows from (A3) and the weak lower semicontinuity of $\varphi$ that

$$
\begin{aligned}
0 & \leq \limsup _{m \rightarrow \infty}\left[F\left(z_{m}, y\right)+\varphi(y)-\varphi\left(z_{m}\right)+\frac{1}{r}\left\langle y-z_{m}, z_{m}-x_{0}\right\rangle\right] \\
& \leq \limsup _{m \rightarrow \infty}\left[F\left(z_{m}, y\right)+\varphi(y)\right]-\liminf _{m \rightarrow \infty} \varphi\left(z_{m}\right)+\frac{1}{r} \limsup _{m \rightarrow \infty}\left\langle y-z_{m}, z_{m}-x_{0}\right\rangle \\
& \leq F(z, y)+\varphi(y)-\varphi(z)+\frac{1}{r}\left\langle z-y, x_{0}-z\right\rangle .
\end{aligned}
$$

This implies that $z \in G(y)$. Hence, $G(y)$ is weakly closed. Hence, $T_{r}\left(x_{0}\right)=$ $\cap_{y \in C} G(y)=\cap_{y \in C} \overline{G(y)}^{w} \neq \emptyset$. Hence, from the arbitrariness of $x_{0}$, we know that $T_{r}(x) \neq \emptyset, \forall x \in H$. We claim that $T_{r}$ is single-valued. Indeed, for $x \in H$ and $r>0$, let $z_{1}, z_{2} \in T_{r}(x)$. Then,

$$
F\left(z_{1}, z_{2}\right)+\varphi\left(z_{2}\right)-\varphi\left(z_{1}\right)+\frac{1}{r}\left\langle z_{2}-z_{1}, z_{1}-x\right\rangle \geq 0
$$

and

$$
F\left(z_{2}, z_{1}\right)+\varphi\left(z_{1}\right)-\varphi\left(z_{2}\right)+\frac{1}{r}\left\langle z_{1}-z_{2}, z_{2}-x\right\rangle \geq 0 .
$$

Adding the two inequalities, we have

$$
F\left(z_{1}, z_{2}\right)+F\left(z_{2}, z_{1}\right)+\frac{1}{r}\left\langle z_{2}-z_{1}, z_{1}-z_{2}\right\rangle \geq 0 .
$$

From (A2) and $r>0$, we have

$$
\left\langle z_{2}-z_{1}, z_{1}-z_{2}\right\rangle \geq 0
$$

So, we have $z_{1}=z_{2}$.

Now we claim that $T_{r}$ is a firmly nonexpansive-type map. Indeed, for $x, y \in H$, we have

$$
F\left(T_{r}(x), T_{r}(y)\right)+\varphi\left(T_{r}(y)\right)-\varphi\left(T_{r}(x)\right)+\frac{1}{r}\left\langle T_{r}(y)-T_{r}(x), T_{r}(x)-x\right\rangle \geq 0
$$

and

$$
F\left(T_{r}(y), T_{r}(x)\right)+\varphi\left(T_{r}(x)\right)-\varphi\left(T_{r}(y)\right)+\frac{1}{r}\left\langle T_{r}(x)-T_{r}(y), T_{r}(y)-y\right\rangle \geq 0 .
$$

Adding the two inequalities, we have

$$
F\left(T_{r}(x), T_{r}(y)\right)+F\left(T_{r}(y), T_{r}(x)\right)+\frac{1}{r}\left\langle T_{r}(y)-T_{r}(x), T_{r}(x)-T_{r}(y)-x+y\right\rangle \geq 0 .
$$

From (A2) and $r>0$, we have

$$
\left\langle T_{r}(y)-T_{r}(x), T_{r}(x)-T_{r}(y)-(x-y)\right\rangle \geq 0 .
$$


Therefore, we have

$$
\left\|T_{r}(x)-T_{r}(y)\right\|^{2} \leq\left\langle T_{r}(x)-T_{r}(y), x-y\right\rangle .
$$

Next we claim that $F i x\left(T_{r}\right)=M E F(F, \varphi)$. Indeed, we have the following:

$$
\begin{aligned}
u \in \operatorname{Fix}\left(T_{r}\right) \Leftrightarrow u & =T_{r}(u) \\
& \Leftrightarrow F(u, y)+\varphi(y)-\varphi(u)+\frac{1}{r}\langle y-u, u-u\rangle \geq 0, \forall y \in C \\
& \Leftrightarrow F(u, y) \geq 0, \forall y \in C \\
& \Leftrightarrow u \in \operatorname{MEF}(F, \varphi) .
\end{aligned}
$$

At last, we claim that $\operatorname{MEF}(F, \varphi)$ is closed convex. Indeed, Since $T_{r}$ is firmly nonexpansive, $T_{r}$ is also nonexpansive. By Lemma 2.2, we know that $\operatorname{MEF}(F, \varphi)=$ $\operatorname{Fix}\left(T_{r}\right)$ is closed and convex.

\section{Remark 2.1.}

(i) Lemma 2.3 generalizes and extends Corollary 5 in [4] and Lemma 2.12 in [6], Lemma 2.1 and 2.2 in [7] which are the foundations for the algorithms of equilibrium problems. And hence Lemma 2.3 plays a key role in the research of algorithms for problems (1.1) and (1.2).

(ii) We observed that in Lemma 3.1 in [1], the condition of the sequentially continuity from the weak topology to the strong topology for the derivative $K$ of the function $K: C \rightarrow R$ is a very strong condition. Even if $K(x)=\frac{\|x\|^{2}}{2}$ and $\eta(x, y)=x-y$, then $K^{\prime}(x)=x$ is not sequentially continuous from the weak topology to the strong topology.

\section{Strong Convergence Theorems}

In this section, we show a strong convergence of an iterative algorithm based on extragradient method and hybrid method which solves the problem of finding a common element of the set of solutions of a generalized mixed equilibrium problem, the set of fixed points of a nonexpansive mapping and the set of the variational inequality for a monotone, Lipschitz-continuous mapping in a Hilbert space.

Theorem 3.1. Let $C$ be a nonempty closed convex subset of a real Hilbert space $H$. Let $F$ be a bifunction from $C \times C$ to $R$ satisfying $(A 1)-(A 5)$ and $\varphi: C \rightarrow R$ be a lower semicontinuous and convex function. Let $A$ be a monotone and $k$-Lipschitz-continuous mapping of $C$ into $H$ and $B$ be an $\alpha$-inverse-strongly monotone mapping of $C$ into $H$. Let $S$ be a nonexpansive mapping of $C$ into $H$ such that $\Omega=\operatorname{Fix}(S) \cap V I(C, A) \cap \operatorname{GMEP}(F, \varphi, B) \neq \emptyset$. Assume that either $(B 1)$ or $(B 2)$ holds. Let $\left\{x_{n}\right\},\left\{u_{n}\right\},\left\{y_{n}\right\}$ and $\left\{z_{n}\right\}$ be sequences generated by 


$$
\left\{\begin{aligned}
x_{1}= & x \in C, \\
& F\left(u_{n}, y\right)+\varphi(y)-\varphi\left(u_{n}\right)+\left\langle B x_{n}, y-u_{n}\right\rangle+\frac{1}{r_{n}}\left\langle y-u_{n}, u_{n}-x_{n}\right\rangle \geq 0, \forall y \in C, \\
y_{n}= & \left(1-\gamma_{n}\right) u_{n}+\gamma_{n} P_{C}\left(u_{n}-\lambda_{n} A u_{n}\right), \\
z_{n}= & \left(1-\alpha_{n}-\beta_{n}\right) x_{n}+\alpha_{n} y_{n}+\beta_{n} S P_{C}\left(u_{n}-\lambda_{n} A y_{n}\right), \\
C_{n}= & \left\{z \in C:\left\|z_{n}-z\right\|^{2} \leq\left\|x_{n}-z\right\|^{2}+\left(3-3 \gamma_{n}+\alpha_{n}\right) b^{2}\left\|A u_{n}\right\|^{2}\right\} \\
Q_{n}= & \left\{z \in C:\left\langle x_{n}-z, x-x_{n}\right\rangle \geq 0\right\}, x_{n+1}=P_{C_{n} \cap Q_{n}} x
\end{aligned}\right.
$$

for every $n=1,2, \ldots$. where $\left\{\lambda_{n}\right\} \subset[a, b]$ for some $[a, b] \subset\left(0, \frac{1}{4 k}\right),\left\{r_{n}\right\} \subset$ $[d, e]$ for some $d, e \in(0,2 \alpha)$, and $\left\{\alpha_{n}\right\},\left\{\beta_{n}\right\},\left\{\gamma_{n}\right\}$ are three sequences in $[0,1]$ satisfying the conditions:

(i) $\alpha_{n}+\beta_{n} \leq 1$ for all $n \in N$;

(ii) $\lim _{n \rightarrow \infty} \alpha_{n}=0$;

(iii) $\liminf _{n \rightarrow \infty} \beta_{n}>0$;

(iii) $\lim _{n \rightarrow \infty} \gamma_{n}=1$ and $\gamma_{n}>\frac{3}{4}$ for all $n \in N$;

Then, $\left\{x_{n}\right\},\left\{u_{n}\right\},\left\{y_{n}\right\}$ and $\left\{z_{n}\right\}$ converge strongly to $w=P_{\Omega}(x)$.

Proof. It is obvious that $C_{n}$ is closed and $Q_{n}$ is closed and convex for every $n=1,2, \ldots$. Since

$$
C_{n}=\left\{z \in H:\left\|z_{n}-x_{n}\right\|^{2}+2\left\langle z_{n}-x_{n}, x_{n}-z\right\rangle \leq\left(3-3 \gamma_{n}+\alpha_{n}\right) b^{2}\left\|A u_{n}\right\|^{2}\right\},
$$

we also have that $C_{n}$ is convex for every $n=1,2, \ldots$ It is easy to see that $\left\langle x_{n}-z, x-x_{n}\right\rangle \geq 0$ for all $z \in Q_{n}$ and by $(2.1), x_{n}=P_{Q_{n}} x$. Put $t_{n}=$ $P_{C}\left(u_{n}-\lambda_{n} A y_{n}\right)$ for every $n=1,2, \ldots$. Let $u \in \Omega$ and let $\left\{T_{r_{n}}\right\}$ be a sequence of mappings defined as in Lemma 2.3. Then $u=P_{C}\left(u-\lambda_{n} A u\right)=T_{r_{n}}\left(u-r_{n} B u\right)$. From $u_{n}=T_{r_{n}}\left(x_{n}-r_{n} B x_{n}\right) \in C$ and the $\alpha$-inverse-strongly monotonicity of $B$, we have

$$
\begin{aligned}
& \left\|u_{n}-u\right\|^{2}=\left\|T_{r_{n}}\left(x_{n}-r_{n} B x_{n}\right)-T_{r_{n}}\left(u-r_{n} B u\right)\right\|^{2} \\
\leq & \left\|x_{n}-r_{n} B x_{n}-\left(u-r_{n} B u\right)\right\|^{2} \\
\leq & \left\|x_{n}-u\right\|^{2}-2 r_{n}\left\langle x_{n}-u, B x_{n}-B u\right\rangle+r_{n}^{2}\left\|B x_{n}-B u\right\|^{2} \\
\leq & \left\|x_{n}-u\right\|^{2}-2 r_{n} \alpha\left\|B x_{n}-B u\right\|^{2}+r_{n}^{2}\left\|B x_{n}-B u\right\|^{2} \\
= & \left\|x_{n}-u\right\|^{2}+r_{n}\left(r_{n}-2 \alpha\right)\left\|B x_{n}-B u\right\|^{2} \\
\leq & \left\|x_{n}-u\right\| .
\end{aligned}
$$


From (2.2), the monotonicity of $A$, and $u \in V I(C, A)$, we have

$$
\begin{aligned}
& \left\|t_{n}-u\right\|^{2} \leq\left\|u_{n}-\lambda_{n} A y_{n}-u\right\|^{2}-\left\|u_{n}-\lambda_{n} A y_{n}-t_{n}\right\|^{2} \\
= & \left\|u_{n}-u\right\|^{2}-\left\|u_{n}-t_{n}\right\|^{2}+2 \lambda_{n}\left\langle A y_{n}, u-t_{n}\right\rangle \\
= & \left\|u_{n}-u\right\|^{2}-\left\|u_{n}-t_{n}\right\|^{2}+2 \lambda_{n}\left(\left\langle A y_{n}-A u, u-y_{n}\right\rangle\right. \\
& \left.+\left\langle A u, u-y_{n}\right\rangle+\left\langle A y_{n}, y_{n}-t_{n}\right\rangle\right) \\
\leq & \left\|u_{n}-u\right\|^{2}-\left\|u_{n}-t_{n}\right\|^{2}+2 \lambda_{n}\left\langle A y_{n}, y_{n}-t_{n}\right\rangle \\
\leq & \left\|u_{n}-u\right\|^{2}-\left\|u_{n}-y_{n}\right\|^{2}-2\left\langle u_{n}-y_{n}, y_{n}-t_{n}\right\rangle \\
& -\left\|y_{n}-t_{n}\right\|^{2}+2 \lambda_{n}\left\langle A y_{n}, y_{n}-t_{n}\right\rangle \\
= & \left\|u_{n}-u\right\|^{2}-\left\|u_{n}-y_{n}\right\|^{2}-\left\|y_{n}-t_{n}\right\|^{2}+2\left\langle u_{n}-\lambda_{n} A y_{n}-y_{n}, t_{n}-y_{n}\right\rangle .
\end{aligned}
$$

Further, Since $y_{n}=\left(1-\gamma_{n}\right) u_{n}+\gamma_{n} P_{C}\left(u_{n}-\lambda_{n} A u_{n}\right)$ and $A$ is $k$-Lipschitzcontinuous, we have

$$
\begin{aligned}
& \left\langle u_{n}-\lambda_{n} A y_{n}-y_{n}, t_{n}-y_{n}\right\rangle \\
= & \left\langle u_{n}-\lambda_{n} A u_{n}-y_{n}, t_{n}-y_{n}\right\rangle+\left\langle\lambda_{n} A u_{n}-\lambda_{n} A y_{n}, t_{n}-y_{n}\right\rangle \\
\leq & \left\langle u_{n}-\lambda_{n} A u_{n}-\left(1-\gamma_{n}\right) u_{n}-\gamma_{n} P_{C}\left(u_{n}-\lambda_{n} A u_{n}\right), t_{n}-y_{n}\right\rangle \\
& +\lambda_{n}\left\|A u_{n}-A y_{n}\right\|\left\|t_{n}-y_{n}\right\| \\
\leq & \gamma_{n}\left\langle u_{n}-\lambda_{n} A u_{n}-P_{C}\left(u_{n}-\lambda_{n} A u_{n}\right), t_{n}-y_{n}\right\rangle \\
& -\left(1-\gamma_{n}\right) \lambda_{n}\left\langle A u_{n}, t_{n}-y_{n}\right\rangle+\lambda_{n} k\left\|u_{n}-y_{n}\right\|\left\|t_{n}-y_{n}\right\| .
\end{aligned}
$$

In addition, from the definition of $P_{C}$, we have

$$
\begin{aligned}
& \left\langle u_{n}-\lambda_{n} A u_{n}-P_{C}\left(u_{n}-\lambda_{n} A u_{n}\right), t_{n}-y_{n}\right\rangle \\
= & \left\langle u_{n}-\lambda_{n} A u_{n}-P_{C}\left(u_{n}-\lambda_{n} A u_{n}\right), t_{n}-\left(1-\gamma_{n}\right) u_{n}-\gamma_{n} P_{C}\left(u_{n}-\lambda_{n} A u_{n}\right)\right\rangle \\
= & \left(1-\gamma_{n}\right)\left\langle u_{n}-\lambda_{n} A u_{n}-P_{C}\left(u_{n}-\lambda_{n} A u_{n}\right), t_{n}-u_{n}\right\rangle \\
& +\gamma_{n}\left\langle u_{n}-\lambda_{n} A u_{n}-P_{C}\left(u_{n}-\lambda_{n} A u_{n}\right), t_{n}-P_{C}\left(u_{n}-\lambda_{n} A u_{n}\right)\right\rangle \\
\leq & \left(1-\gamma_{n}\right)\left\|u_{n}-\lambda_{n} A u_{n}-P_{C}\left(u_{n}-\lambda_{n} A u_{n}\right)\right\|\left\|t_{n}-u_{n}\right\| \\
\leq & \left(1-\gamma_{n}\right) \lambda_{n}\left\|u_{n}-A u_{n}-u_{n}\right\|\left(\left\|t_{n}-y_{n}\right\|+\left\|y_{n}-u_{n}\right\|\right) \\
\leq & \left(1-\gamma_{n}\right) \lambda_{n}\left\|A u_{n}\right\|\left(\left\|t_{n}-y_{n}\right\|+\left\|y_{n}-u_{n}\right\|\right) .
\end{aligned}
$$

So, from the assumptions $b<\frac{1}{4 k}, \gamma_{n}>\frac{3}{4}$ and (3.1), we have 


$$
\begin{aligned}
& \left\|t_{n}-u\right\|^{2} \leq\left\|u_{n}-u\right\|^{2}-\left\|u_{n}-y_{n}\right\|^{2}-\left\|y_{n}-t_{n}\right\|^{2} \\
& +2 \gamma_{n}\left(1-\gamma_{n}\right) b\left\|A u_{n}\right\|\left(\left\|t_{n}-y_{n}\right\|+\left\|y_{n}-u_{n}\right\|\right) \\
& +2\left(1-\gamma_{n}\right) b\left\|A u_{n}\right\|\left\|t_{n}-y_{n}\right\|+2 b k\left\|u_{n}-y_{n}\right\|\left\|t_{n}-y_{n}\right\| \\
\leq & \left\|u_{n}-u\right\|^{2}-\left\|u_{n}-y_{n}\right\|^{2}-\left\|y_{n}-t_{n}\right\|^{2}+\left(1-\gamma_{n}\right)\left(2 b^{2}\left\|A u_{n}\right\|^{2}\right. \\
& \left.+\left\|t_{n}-y_{n}\right\|^{2}+\left\|y_{n}-u_{n}\right\|^{2}\right)+\left(1-\gamma_{n}\right)\left(b^{2}\left\|A u_{n}\right\|^{2}\right. \\
& \left.+\left\|t_{n}-y_{n}\right\|^{2}\right)+b k\left(\left\|u_{n}-y_{n}\right\|^{2}+\left\|t_{n}-y_{n}\right\|^{2}\right) \\
= & \left\|u_{n}-u\right\|^{2}-\left(\gamma_{n}-b k\right)\left\|u_{n}-y_{n}\right\|^{2} \\
+ & \left(1-2 \gamma_{n}+b k\right)\left\|t_{n}-y_{n}\right\|^{2}+3\left(1-\gamma_{n}\right) b^{2}\left\|A u_{n}\right\|^{2} \\
\leq & \left\|u_{n}-u\right\|^{2}+3\left(1-\gamma_{n}\right) b^{2}\left\|A u_{n}\right\|^{2} \\
\leq & \left\|x_{n}-u\right\|^{2}+3\left(1-\gamma_{n}\right) b^{2}\left\|A u_{n}\right\|^{2} .
\end{aligned}
$$

In addition, from $u \in V I(C, A)$ and (3.1), we have

$$
\begin{aligned}
& \left\|y_{n}-u\right\|^{2}=\left\|\left(1-\gamma_{n}\right)\left(u_{n}-u\right)+\gamma_{n}\left(P_{C}\left(u_{n}-\lambda_{n} A u_{n}\right)-u\right)\right\|^{2} \\
\leq & \left(1-\gamma_{n}\right)\left\|u_{n}-u\right\|^{2}+\gamma_{n}\left\|P_{C}\left(u_{n}-\lambda_{n} A u_{n}\right)-P_{C}(u)\right\|^{2} \\
\leq & \left(1-\gamma_{n}\right)\left\|u_{n}-u\right\|^{2}+\gamma_{n}\left\|u_{n}-\lambda_{n} A u_{n}-u\right\|^{2} \\
\leq & \left(1-\gamma_{n}\right)\left\|u_{n}-u\right\|^{2}+\gamma_{n}\left[\left\|u_{n}-u\right\|^{2}-2 \lambda_{n}\left\langle A u_{n}, u_{n}-u\right\rangle+\lambda_{n}^{2}\left\|A u_{n}\right\|^{2}\right] \\
\leq & \left\|u_{n}-u\right\|^{2}+b^{2}\left\|A u_{n}\right\|^{2} \\
\leq & \left\|x_{n}-u\right\|^{2}+b^{2}\left\|A u_{n}\right\|^{2} .
\end{aligned}
$$

Therefore from (3.1)- (3.3), $z_{n}=\left(1-\alpha_{n}-\beta_{n}\right) x_{n}+\alpha_{n} y_{n}+\beta_{n} S t_{n}$ and $u=S u$, we have

$$
\begin{aligned}
& \left\|z_{n}-u\right\|^{2}=\left\|\left(1-\alpha_{n}-\beta_{n}\right) x_{n}+\alpha_{n} y_{n}+\beta_{n} S t_{n}-u\right\|^{2} \\
\leq & \left(1-\alpha_{n}-\beta_{n}\right)\left\|x_{n}-u\right\|^{2}+\alpha_{n}\left\|y_{n}-u\right\|^{2}+\beta_{n}\left\|S t_{n}-u\right\|^{2} \\
\leq & \left(1-\alpha_{n}-\beta_{n}\right)\left\|x_{n}-u\right\|^{2}+\alpha_{n}\left\|y_{n}-u\right\|^{2}+\beta_{n}\left\|t_{n}-u\right\|^{2} \\
\leq & \left(1-\alpha_{n}-\beta_{n}\right)\left\|x_{n}-u\right\|^{2}+\alpha_{n}\left[\left\|u_{n}-u\right\|^{2}+b^{2}\left\|A u_{n}\right\|^{2}\right] \\
& +\beta_{n}\left[\left\|u_{n}-u\right\|^{2}+3\left(1-\gamma_{n}\right) b^{2}\left\|A u_{n}\right\|^{2}\right] \\
\leq & \left.\left\|x_{n}-u\right\|^{2}+\left(3-3 \gamma_{n}+\alpha_{n}\right) b^{2}\left\|A u_{n}\right\|^{2}\right],
\end{aligned}
$$

for every $n=1,2, \ldots$ and hence $u \in C_{n}$. So, $\Omega \subset C_{n}$ for every $n=1,2, \ldots$ Next, let us show by mathematical induction that $\left\{x_{n}\right\}$ is well defined and $\Omega \subset C_{n} \cap Q_{n}$ for every $n=1,2, \ldots$. For $n=1$ we have $x_{1}=x \in C$ and $Q_{1}=C$. Hence we obtain $\Omega \subset C_{1} \cap Q_{1}$. Suppose that $x_{k}$ is given and $\Omega \subset C_{k} \cap Q_{k}$ for some 
$k \in N$. Since $\Omega$ is nonempty, $C_{k} \cap Q_{k}$ is a nonempty closed convex subset of $H$. So, there exists a unique element $x_{k+1} \in C_{k} \cap Q_{k}$ such that $x_{k+1}=P_{C_{k} \cap Q_{k}} x$. It is also obvious that there holds $\left\langle x_{k+1}-z, x-x_{k+1}\right\rangle \geq 0$ for every $z \in C_{k} \cap Q_{k}$. Since $\Omega \subset C_{k} \cap Q_{k}$, we have $\left\langle x_{k+1}-z, x-x_{k+1}\right\rangle \geq 0$ for every $z \in \Omega$ and hence $\Omega \subset Q_{k+1}$. Therefore, we obtain $\Omega \subset C_{k+1} \cap Q_{k+1}$.

Let $l_{0}=P_{\Omega} x$. From $x_{n+1}=P_{C_{n} \cap Q_{n}} x$ and $l_{0} \in \Omega \subset C_{n} \cap Q_{n}$, we have

$$
\left\|x_{n+1}-x\right\| \leq\left\|l_{0}-x\right\|
$$

for every $n=1,2, \ldots$. Therefore, $\left\{x_{n}\right\}$ is bounded. From (3.1)-(3.4) and the lipschitz continuity of $A$, we also obtain that $\left\{u_{n}\right\},\left\{A u_{n}\right\},\left\{t_{n}\right\}$ and $\left\{z_{n}\right\}$ are bounded. Since $x_{n+1} \in C_{n} \cap Q_{n} \subset C_{n}$ and $x_{n}=P_{Q_{n}} x$, we have

$$
\left\|x_{n}-x\right\| \leq\left\|x_{n+1}-x\right\|
$$

for every $n=1,2, \ldots$ It follows from (3.5) that $\lim _{n \rightarrow \infty}\left\|x_{n}-x\right\|$ exists.

Since $x_{n}=P_{Q_{n}} x$ and $x_{n+1} \in Q_{n}$, using (2.2), we have

$$
\left\|x_{n+1}-x_{n}\right\|^{2} \leq\left\|x_{n+1}-x\right\|^{2}-\left\|x_{n}-x\right\|^{2}
$$

for every $n=1,2, \ldots$. This implies that

$$
\lim _{n \rightarrow \infty}\left\|x_{n+1}-x_{n}\right\|=0
$$

Since $x_{n+1} \in C_{n}$, we have $\left\|z_{n}-x_{n+1}\right\|^{2} \leq\left\|x_{n}-x_{n+1}\right\|^{2}+\left(3-3 \gamma_{n}+\alpha_{n}\right) b^{2}\left\|A u_{n}\right\|^{2}$ and hence it follows from $\lim _{n \rightarrow \infty} \gamma_{n}=1$ and $\lim _{n \rightarrow \infty} \alpha_{n}=0$ that $\lim _{n \rightarrow \infty} \| z_{n}-$ $x_{n+1} \|=0$. Since

$$
\left\|x_{n}-z_{n}\right\| \leq\left\|x_{n}-x_{n+1}\right\|+\left\|x_{n+1}-z_{n}\right\|
$$

for every $n=1,2, \ldots$, we have $\left\|x_{n}-z_{n}\right\| \rightarrow 0$.

For $u \in \Omega$, from (3.4) we obtain

$$
\begin{aligned}
& \left\|z_{n}-u\right\|^{2}-\left\|x_{n}-u\right\|^{2} \\
\leq & \left(-\alpha_{n}-\beta_{n}\right)\left\|x_{n}-u\right\|^{2}+\alpha_{n}\left\|y_{n}-u\right\|^{2}+\beta_{n}\left\|S t_{n}-u\right\|^{2} \\
\leq & \left(3-3 \gamma_{n}+\alpha_{n}\right) b^{2}\left\|A u_{n}\right\|^{2} .
\end{aligned}
$$

Since $\lim _{n \rightarrow \infty} \gamma_{n}=1$ and $\lim _{n \rightarrow \infty} \alpha_{n}=0,\left\{u_{n}\right\},\left\{A u_{n}\right\}$ and $\left\{z_{n}\right\}$ are bounded, we have

$$
\lim _{n \rightarrow \infty} \beta_{n}\left(\left\|S t_{n}-u\right\|^{2}-\left\|x_{n}-u\right\|^{2}\right)=0 .
$$

By $\liminf _{n \rightarrow \infty} \beta_{n}>0$, we get

$$
\lim _{n \rightarrow \infty}\left\|S t_{n}-u\right\|^{2}-\left\|x_{n}-u\right\|^{2}=0
$$


From (3.2) and $u=S u$, we have

$$
\begin{aligned}
& \lim _{n \rightarrow \infty}\left\|S t_{n}-u\right\|^{2}-\left\|x_{n}-u\right\|^{2} \leq \lim _{n \rightarrow \infty}\left\|t_{n}-u\right\|^{2}-\left\|x_{n}-u\right\|^{2} \\
\leq & \lim _{n \rightarrow \infty} 3\left(1-\gamma_{n}\right) b^{2}\left\|A u_{n}\right\|^{2}=0 .
\end{aligned}
$$

Thus, $\lim _{n \rightarrow \infty}\left\|t_{n}-u\right\|^{2}-\left\|x_{n}-u\right\|^{2}=0$.

From (3.2) and (3.1), we have

$$
\begin{aligned}
& \left(\gamma_{n}-b k\right)\left\|u_{n}-y_{n}\right\|^{2}+\left(2 \gamma_{n}-1-b k\right)\left\|t_{n}-y_{n}\right\|^{2} \\
\leq & \left\|x_{n}-u\right\|^{2}-\left\|t_{n}-u\right\|^{2}+3\left(1-\gamma_{n}\right) b^{2}\left\|A u_{n}\right\|^{2} .
\end{aligned}
$$

It follows that

$$
\lim _{n \rightarrow \infty}\left(\gamma_{n}-b k\right)\left\|u_{n}-y_{n}\right\|^{2}+\left(2 \gamma_{n}-1-b k\right)\left\|t_{n}-y_{n}\right\|^{2}=0 .
$$

The assumptions on $\gamma_{n}$ and $\lambda_{n}$ imply that $\gamma_{n}-b k>\frac{1}{2}$ and $2 \gamma_{n}-1-b k>\frac{1}{4}$. Consequently, $\lim _{n \rightarrow \infty}\left\|u_{n}-y_{n}\right\|=\lim _{n \rightarrow \infty}\left\|t_{n}-y_{n}\right\|=0$. Since $A$ is Lipschitz continuous, we have $\lim _{n \rightarrow \infty}\left\|A t_{n}-A y_{n}\right\|=0$. It follows from $\left\|u_{n}-t_{n}\right\| \leq$ $\left\|u_{n}-y_{n}\right\|+\left\|t_{n}-y_{n}\right\|$ that $\lim _{n \rightarrow \infty}\left\|u_{n}-t_{n}\right\|=0$.

We rewrite the definition of $z_{n}$ as

$$
z_{n}-x_{n}=\alpha_{n}\left(y_{n}-x_{n}\right)+\beta_{n}\left(S t_{n}-x_{n}\right) .
$$

From $\lim _{n \rightarrow \infty}\left\|z_{n}-x_{n}\right\|=0, \lim _{n \rightarrow \infty} \alpha_{n}=0$, the boundedness of $\left\{x_{n}\right\},\left\{y_{n}\right\}$ and $\liminf _{n \rightarrow \infty} \beta_{n}>0$ we infer that $\lim _{n \rightarrow \infty}\left\|S t_{n}-x_{n}\right\|=0$.

By (3.4) and (3.1), we have

$$
\begin{aligned}
& \left\|z_{n}-u\right\|^{2} \leq\left(1-\alpha_{n}-\beta_{n}\right)\left\|x_{n}-u\right\|^{2}+\alpha_{n}\left[\left\|u_{n}-u\right\|^{2}\right. \\
& \left.+b^{2}\left\|A u_{n}\right\|^{2}\right]+\beta_{n}\left[\left\|u_{n}-u\right\|^{2}+3\left(1-\gamma_{n}\right) b^{2}\left\|A u_{n}\right\|^{2}\right] \\
\leq & \left(1-\alpha_{n}-\beta_{n}\right)\left\|x_{n}-u\right\|^{2}+\alpha_{n}\left[\left\|x_{n}-u\right\|^{2}\right. \\
& \left.+r_{n}\left(r_{n}-2 \alpha\right)\left\|B x_{n}-B u\right\|^{2}+b^{2}\left\|A u_{n}\right\|^{2}\right] \\
& +\beta_{n}\left[\left\|x_{n}-u\right\|^{2}+r_{n}\left(r_{n}-2 \alpha\right)\left\|B x_{n}-B u\right\|^{2}+3\left(1-\gamma_{n}\right) b^{2}\left\|A u_{n}\right\|^{2}\right] \\
\leq & \left\|x_{n}-u\right\|^{2}+\left(\alpha_{n}+\beta_{n}\right) r_{n}\left(r_{n}-2 \alpha\right)\left\|B x_{n}-B u\right\|^{2} \\
& \left.+\left(3-3 \gamma_{n}+\alpha_{n}\right) b^{2}\left\|A u_{n}\right\|^{2}\right] .
\end{aligned}
$$

Hence, we have

$$
\begin{aligned}
& \left(\alpha_{n}+\beta_{n}\right) d(2 \alpha-e)\left\|B x_{n}-B u\right\|^{2} \\
\leq & \left(\alpha_{n}+\beta_{n}\right) r_{n}\left(2 \alpha-r_{n}\right)\left\|B x_{n}-B u\right\|^{2} \\
\leq & \left\|x_{n}-u\right\|^{2}-\left\|z_{n}-u\right\|^{2}+\left(3-3 \gamma_{n}+\alpha_{n}\right) b^{2}\left\|A u_{n}\right\|^{2} \\
\leq & \left(\left\|x_{n}-u\right\|+\left\|z_{n}-u\right\|\right)\left\|x_{n}-z_{n}\right\|+\left(3-3 \gamma_{n}+\alpha_{n}\right) b^{2}\left\|A u_{n}\right\|^{2} .
\end{aligned}
$$


Since $\lim _{n \rightarrow \infty} \alpha_{n}=0, \liminf _{n \rightarrow \infty} \beta_{n}>0, \lim _{n \rightarrow \infty} \gamma_{n}=1,\left\|x_{n}-z_{n}\right\| \rightarrow 0$ and the sequences $\left\{x_{n}\right\}$ and $\left\{z_{n}\right\}$ are bounded, we obtain $\left\|B x_{n}-B u\right\| \rightarrow 0$.

For $u \in \Omega$, we have, from Lemma 2.3,

$$
\begin{aligned}
& \left\|u_{n}-u\right\|^{2}=\left\|T_{r_{n}}\left(x_{n}-r_{n} B x_{n}\right)-T_{r_{n}}\left(u-r_{n} B u\right)\right\|^{2} \\
\leq & \left\langle T_{r_{n}}\left(x_{n}-r_{n} B x_{n}\right)-T_{r_{n}}\left(u-r_{n} B u\right), x_{n}-r_{n} B x_{n}-\left(u-r_{n} B u\right)\right\rangle \\
= & \frac{1}{2}\left\{\left\|u_{n}-u\right\|^{2}+\left\|x_{n}-r_{n} B x_{n}-\left(u-r_{n} B u\right)\right\|^{2}\right. \\
& \left.-\left\|x_{n}-r_{n} B x_{n}-\left(u-r_{n} B u\right)-\left(u_{n}-u\right)\right\|^{2}\right\} \\
\leq & \frac{1}{2}\left\{\left\|u_{n}-u\right\|^{2}+\left\|x_{n}-u\right\|^{2}-\left\|x_{n}-r_{n} B x_{n}-\left(u-r_{n} B u\right)-\left(u_{n}-u\right)\right\|^{2}\right\} \\
= & \frac{1}{2}\left\{\left\|u_{n}-u\right\|^{2}+\left\|x_{n}-u\right\|^{2}-\left\|x_{n}-u_{n}\right\|^{2}\right. \\
& +2 r_{n}\left\langle B x_{n}-B u, x_{n}-u_{n}\right\rangle-r_{n}^{2}\left\|B x_{n}-B u\right\|^{2} .
\end{aligned}
$$

Hence,

$$
\begin{gathered}
\left\|u_{n}-u\right\|^{2} \leq\left\|x_{n}-u\right\|^{2}-\left\|x_{n}-u_{n}\right\|^{2}+2 r_{n}\left\langle B x_{n}-B u, x_{n}-u_{n}\right\rangle \\
-r_{n}^{2}\left\|B x_{n}-B u\right\|^{2} \leq\left\|x_{n}-u\right\|^{2}-\left\|x_{n}-u_{n}\right\|^{2}+2 r_{n}\left\langle B x_{n}-B u, x_{n}-u_{n}\right\rangle .
\end{gathered}
$$

Then, by (3.4), we have

$$
\begin{aligned}
& \left\|z_{n}-u\right\|^{2} \leq\left(1-\alpha_{n}-\beta_{n}\right)\left\|x_{n}-u\right\|^{2}+\alpha_{n}\left[\left\|u_{n}-u\right\|^{2}+b^{2}\left\|A u_{n}\right\|^{2}\right] \\
& +\beta_{n}\left[\left\|u_{n}-u\right\|^{2}+3\left(1-\gamma_{n}\right) b^{2}\left\|A u_{n}\right\|^{2}\right] \\
\leq & \left(1-\alpha_{n}-\beta_{n}\right)\left\|x_{n}-u\right\|^{2}+\alpha_{n}\left[\left(\left\|x_{n}-u\right\|^{2}-\left\|x_{n}-u_{n}\right\|^{2}\right.\right. \\
& \left.\left.+2 r_{n}\left\langle B x_{n}-B u, x_{n}-u_{n}\right\rangle\right)+b^{2}\left\|A u_{n}\right\|^{2}\right]+\beta_{n}\left[\left(\left\|x_{n}-u\right\|^{2}\right.\right. \\
& \left.\left.-\left\|x_{n}-u_{n}\right\|^{2}+2 r_{n}\left\langle B x_{n}-B u, x_{n}-u_{n}\right\rangle\right)+3\left(1-\gamma_{n}\right) b^{2}\left\|A u_{n}\right\|^{2}\right] \\
\leq & \left\|x_{n}-u\right\|^{2}+\left(-\alpha_{n}-\beta_{n}\right)\left\|x_{n}-u_{n}\right\|^{2}+2 r_{n}\left\|B x_{n}-B u\right\|\left\|x_{n}-u_{n}\right\| \\
& +\left(3-3 \gamma_{n}+\alpha_{n}\right) b^{2}\left\|A u_{n}\right\|^{2}
\end{aligned}
$$

Hence,

$$
\begin{aligned}
& \left(\alpha_{n}+\beta_{n}\right)\left\|x_{n}-u_{n}\right\|^{2} \leq\left\|x_{n}-u\right\|^{2}-\left\|z_{n}-u\right\|^{2} \\
& +2 r_{n}\left\|B x_{n}-B u\right\|\left\|x_{n}-u_{n}\right\|+\left(3-3 \gamma_{n}+\alpha_{n}\right) b^{2}\left\|A u_{n}\right\|^{2} \\
\leq & \left(\left\|x_{n}-u\right\|+\left\|z_{n}-u\right\|\right)\left\|x_{n}-z_{n}\right\| \\
& +2 r_{n}\left\|B x_{n}-B u\right\|\left\|x_{n}-u_{n}\right\|+\left(3-3 \gamma_{n}+\alpha_{n}\right) b^{2}\left\|A u_{n}\right\|^{2} .
\end{aligned}
$$

Since $\lim _{n \rightarrow \infty} \alpha_{n}=0, \liminf _{n \rightarrow \infty} \beta_{n}>0, \lim _{n \rightarrow \infty} \gamma_{n}=1,\left\|x_{n}-z_{n}\right\| \rightarrow 0$, $\left\|B x_{n}-B u\right\| \rightarrow 0$ and the sequences $\left\{x_{n}\right\}$ and $\left\{z_{n}\right\}$ are bounded, we obtain 
$\left\|x_{n}-u_{n}\right\| \rightarrow 0$. From $\left\|z_{n}-t_{n}\right\| \leq\left\|z_{n}-x_{n}\right\|+\left\|x_{n}-u_{n}\right\|+\left\|u_{n}-t_{n}\right\|$ we have $\left\|z_{n}-t_{n}\right\| \rightarrow 0$. From $\left\|t_{n}-x_{n}\right\| \leq\left\|t_{n}-u_{n}\right\|+\left\|x_{n}-u_{n}\right\|$ we also have $\left\|t_{n}-x_{n}\right\| \rightarrow 0$.

Since $z_{n}=\left(1-\alpha_{n}-\beta_{n}\right) x_{n}+\alpha_{n} y_{n}+\beta_{n} S t_{n}$, we have $\beta_{n}\left(S t_{n}-t_{n}\right)=$ $\left(1-\alpha_{n}-\beta_{n}\right)\left(t_{n}-x_{n}\right)+\alpha_{n}\left(t_{n}-y_{n}\right)+\left(z_{n}-t_{n}\right)$. Then

$$
\beta_{n}\left\|S t_{n}-t_{n}\right\| \leq\left(1-\alpha_{n}-\beta_{n}\right)\left\|t_{n}-x_{n}\right\|+\alpha_{n}\left\|t_{n}-y_{n}\right\|+\left\|z_{n}-t_{n}\right\|
$$

and hence $\left\|S t_{n}-t_{n}\right\| \rightarrow 0$.

As $\left\{x_{n}\right\}$ is bounded, there exists a subsequence $\left\{x_{n i}\right\}$ of $\left\{x_{n}\right\}$ such that $x_{n i} \rightarrow$ $w$. From $\left\|x_{n}-u_{n}\right\| \rightarrow 0$, we obtain that $u_{n i} \rightarrow w$. From $\left\|u_{n}-t_{n}\right\| \rightarrow 0$, we also obtain that $t_{n i} \rightarrow w$. Since $\left\{u_{n i}\right\} \subset C$ and $C$ is closed and convex, we obtain $w \in C$.

First, we show $w \in G M E P(F, \varphi, B)$. By $u_{n}=T_{r_{n}}\left(x_{n}-r_{n} B x_{n}\right)$, we know that

$$
F\left(u_{n}, y\right)+\varphi(y)-\varphi\left(u_{n}\right)+\left\langle B x_{n}, y-u_{n}\right\rangle+\frac{1}{r_{n}}\left\langle y-u_{n}, u_{n}-x_{n}\right\rangle \geq 0, \forall y \in C .
$$

It follows from (A2) that

$$
\varphi(y)-\varphi\left(u_{n}\right)+\left\langle B x_{n}, y-u_{n}\right\rangle+\frac{1}{r_{n}}\left\langle y-u_{n}, u_{n}-x_{n}\right\rangle \geq F\left(y, u_{n}\right), \forall y \in C .
$$

Hence,

$$
\varphi(y)-\varphi\left(u_{n_{i}}\right)+\left\langle B x_{n_{i}}, y-u_{n_{i}}\right\rangle+\left\langle y-u_{n_{i}}, \frac{u_{n_{i}}-x_{n_{i}}}{r_{n_{i}}}\right\rangle \geq F\left(y, u_{n_{i}}\right), \forall y \in C
$$

For $t$ with $0<t \leq 1$ and $y \in C$, let $y_{t}=t y+(1-t) w$. Since $y \in C$ and $w \in C$, we obtain $y_{t} \in C$. So, from (3.7) we have

$$
\begin{aligned}
\left\langle y_{t}-u_{n_{i}}, B y_{t}\right\rangle \geq & \left\langle y_{t}-u_{n_{i}}, B y_{t}\right\rangle-\varphi\left(y_{t}\right)+\varphi\left(u_{n_{i}}\right)-\left\langle y_{t}-u_{n_{i}}, B x_{n_{i}}\right\rangle \\
& -\left\langle y_{t}-u_{n_{i}}, \frac{u_{n_{i}}-x_{n_{i}}}{r_{n_{i}}}+F\left(y_{t}, u_{n_{i}}\right)\right. \\
= & \left\langle y_{t}-u_{n_{i}}, B y_{t}-B u_{n_{i}}\right\rangle+\left\langle y_{t}-u_{n_{i}}, B u_{n_{i}}-B x_{n_{i}}\right\rangle-\varphi\left(y_{t}\right)+\varphi\left(u_{n_{i}}\right) \\
& -\left\langle y_{t}-u_{n_{i}}, \frac{u_{n_{i}}-x_{n_{i}}}{r_{n_{i}}}\right\rangle+F\left(y_{t}, u_{n_{i}}\right) .
\end{aligned}
$$

Since $\left\|u_{n_{i}}-x_{n_{i}}\right\| \rightarrow 0$, we have $\left\|B u_{n_{i}}-B x_{n_{i}}\right\| \rightarrow 0$. Further, from the inverse-strongly monotonicity of $B$, we have $\left\langle y_{t}-u_{n_{i}}, B y_{t}-B u_{n_{i}}\right\rangle \geq 0$. So, from (A4), (A5), and the weakly lower semicontinuity of $\varphi, \frac{u_{n_{i}}-x_{n_{i}}}{r_{n_{i}}} \rightarrow 0$ and $u_{n_{i}} \rightarrow w$, we have

$$
\left\langle y_{t}-w, B y_{t}\right\rangle \geq-\varphi\left(y_{t}\right)+\varphi(w)+F\left(y_{t}, w\right)
$$


as $i \rightarrow \infty$. From (A1), (A4) and (3.8), we also have

$$
\begin{aligned}
0 & =F\left(y_{t}, y_{t}\right)+\varphi\left(y_{t}\right)-\varphi\left(y_{t}\right) \\
& \leq t F\left(y_{t}, y\right)+(1-t) F\left(y_{t}, w\right)+t \varphi(y)+(1-t) \varphi(w)-\varphi\left(y_{t}\right) \\
& =t\left[F\left(y_{t}, y\right)+\varphi(y)-\varphi\left(y_{t}\right)\right]+(1-t)\left[F\left(y_{t}, w\right)+\varphi(w)-\varphi\left(y_{t}\right)\right] \\
& \leq t\left[F\left(y_{t}, y\right)+\varphi(y)-\varphi\left(y_{t}\right)\right]+(1-t)\left\langle y_{t}-w, B y_{t}\right\rangle \\
& =t\left[F\left(y_{t}, y\right)+\varphi(y)-\varphi\left(y_{t}\right)\right]+(1-t) t\left\langle y-w, B y_{t}\right\rangle
\end{aligned}
$$

and hence

$$
0 \leq F\left(y_{t}, y\right)+\varphi(y)-\varphi\left(y_{t}\right)+(1-t)\left\langle y-w, B y_{t}\right\rangle .
$$

Letting $t \rightarrow 0$, we have, for each $y \in C$,

$$
F(w, y)+\varphi(y)-\varphi(w)+\langle y-w, B w\rangle \geq 0 .
$$

This implies that $w \in G M E P(F, \varphi, B)$.

We next show that $w \in F i x(S)$. Assume $w \notin F i x(S)$. Since $t_{n_{i}} \rightarrow w$ and $w \neq S w$, from the Opial theorem [29] we have

$$
\begin{aligned}
\liminf _{i \rightarrow \infty}\left\|t_{n_{i}}-w\right\| & <\liminf _{i \rightarrow \infty}\left\|t_{n_{i}}-S w\right\| \\
& \leq \liminf _{i \rightarrow \infty}\left\{\left\|t_{n_{i}}-S t_{n_{i}}\right\|+\left\|S t_{n_{i}}-S w\right\|\right\} \\
& \leq \liminf _{i \rightarrow \infty}\left\|t_{n_{i}}-w\right\|
\end{aligned}
$$

This is a contradiction. So, we get $w \in F i x(S)$.

Finally we show $w \in V I(C, A)$. Let

$$
T v= \begin{cases}A v+N_{C} v & \text { if } v \in C, \\ \emptyset & \text { if } v \notin C .\end{cases}
$$

where $N_{C} v$ is the normal cone to $C$ at $v \in C$. We have already mentioned that in this case the mapping $T$ is maximal monotone, and $0 \in T v$ if and only if $v \in V I(C, A)$. Let $(v, g) \in G(T)$. Then $T v=A v+N_{C} v$ and hence $g-A v \in N_{C} v$. So, we have $\langle v-t, g-A v\rangle \geq 0$ for all $t \in C$. On the other hand, from $t_{n}=P_{C}\left(u_{n}-\lambda_{n} A y_{n}\right)$ and $v \in C$ we have

$$
\left\langle u_{n}-\lambda_{n} A y_{n}-t_{n}, t_{n}-v\right\rangle \geq 0
$$

and hence

$$
\left\langle v-t_{n}, \frac{t_{n}-u_{n}}{\lambda_{n}}+A y_{n}\right\rangle \geq 0
$$


Therefore, we have

$$
\begin{aligned}
\left\langle v-t_{n_{i}}, g\right\rangle & \geq\left\langle v-t_{n_{i}}, A v\right\rangle \\
& \geq\left\langle v-t_{n_{i}}, A v\right\rangle-\left\langle v-t_{n_{i}}, \frac{t_{n_{i}}-u_{n_{i}}}{\lambda_{n_{i}}}+A y_{n_{i}}\right\rangle \\
& =\left\langle v-t_{n_{i}}, A v-A y_{n_{i}}-\frac{t_{n_{i}}-u_{n_{i}}}{\lambda_{n_{i}}}\right\rangle \\
& =\left\langle v-t_{n_{i}}, A v-A t_{n_{i}}+A t_{n_{i}}-A y_{n_{i}}-\frac{t_{n_{i}}-u_{n_{i}}}{\lambda_{n_{i}}}\right\rangle \\
& =\left\langle v-t_{n_{i}}, A v-A t_{n_{i}}\right\rangle+\left\langle v-t_{n_{i}}, A t_{n_{i}}-A y_{n_{i}}\right\rangle-\left\langle v-t_{n_{i}}, \frac{t_{n_{i}}-u_{n_{i}}}{\lambda_{n_{i}}}\right\rangle \\
& \geq\left\langle v-t_{n_{i}}, A t_{n_{i}}-A y_{n_{i}}\right\rangle-\left\langle v-t_{n_{i}}, \frac{t_{n_{i}}-u_{n_{i}}}{\lambda_{n_{i}}}\right\rangle
\end{aligned}
$$

Hence we obtain $\langle v-w, g\rangle \geq 0$ as $i \rightarrow \infty$. Since $T$ is maximal monotone, we have $w \in T^{-1} 0$ and hence $w \in V I(C, A)$. This implies $w \in \Omega$.

From $l_{0}=P_{\Omega} x, w \in \Omega$ and (3.5), we have

$$
\left\|l_{0}-x\right\| \leq\|w-x\| \leq \liminf _{i \rightarrow \infty}\left\|x_{n_{i}}-x\right\| \leq \limsup _{i \rightarrow \infty}\left\|x_{n_{i}}-x\right\| \leq\left\|l_{0}-x\right\| .
$$

So, we obtain

$$
\lim _{i \rightarrow \infty}\left\|x_{n_{i}}-x\right\|=\|w-x\| .
$$

From $x_{n_{i}}-x \rightarrow w-x$ we have $x_{n_{i}}-x \rightarrow w-x$ and hence $x_{n_{i}} \rightarrow w$. Since $x_{n}=P_{Q_{n}} x$ and $l_{0} \in \Omega \subset C_{n} \cap Q_{n} \subset Q_{n}$, we have

$$
-\left\|l_{0}-x_{n_{i}}\right\|^{2}=\left\langle l_{0}-x_{n_{i}}, x_{n_{i}}-x\right\rangle+\left\langle l_{0}-x_{n_{i}}, x-l_{0}\right\rangle \geq\left\langle l_{0}-x_{n_{i}}, x-l_{0}\right\rangle .
$$

As $i \rightarrow \infty$, we obtain $-\left\|l_{0}-w\right\|^{2} \geq\left\langle l_{0}-w, x-l_{0}\right\rangle \geq 0$ by $l_{0}=P_{\Omega} x$ and $w \in \Omega$. Hence we have $w=l_{0}$. This implies that $x_{n} \rightarrow l_{0}$. It is easy to see $u_{n} \rightarrow l_{0}$, $y_{n} \rightarrow l_{0}$ and $z_{n} \rightarrow l_{0}$. The proof is now complete.

By Theorem 3.1, we can obtain some new and interesting strong convergence theorems for some algorithms of finding the solution of generalized mixed equilibrium problem as follows.

Theorem 3.2. Let $C$ be a nonempty closed convex subset of a real Hilbert space $H$. Let $F$ be a bifunction from $C \times C$ to $R$ satisfying $(A 1)-(A 5)$ and $\varphi: C \rightarrow R$ be a lower semicontinuous and convex function. Let $B$ be an $\alpha$-inverse-strongly monotone mapping of $C$ into $H$. Let $S$ be a nonexpansive mapping of $C$ into $H$ such that Fix $(S) \cap G M E P(F, \varphi, B) \neq \emptyset$. Assume that either $(B 1)$ or $(B 2)$ holds. Let $\left\{x_{n}\right\},\left\{u_{n}\right\}$ and $\left\{z_{n}\right\}$ be sequences generated by 


$$
\left\{\begin{array}{l}
x_{1}=x \in C, \\
F\left(u_{n}, y\right)+\varphi(y)-\varphi\left(u_{n}\right)+\left\langle B x_{n}, y-u_{n}\right\rangle+\frac{1}{r_{n}}\left\langle y-u_{n}, u_{n}-x_{n}\right\rangle \geq 0, \quad \forall y \in C, \\
z_{n}=\left(1-\alpha_{n}-\beta_{n}\right) x_{n}+\alpha_{n} u_{n}+\beta_{n} S u_{n}, \\
C_{n}=\left\{z \in C:\left\|z_{n}-z\right\| \leq\left\|x_{n}-z\right\|\right\}, \\
Q_{n}=\left\{z \in C:\left\langle x_{n}-z, x-x_{n}\right\rangle \geq 0\right\}, \\
x_{n+1}=P_{C_{n} \cap Q_{n}} x
\end{array}\right.
$$

for every $n=1,2, \ldots$. where $\left\{r_{n}\right\} \subset[d, e]$ for some $d, e \in(0,2 \alpha)$, and $\left\{\alpha_{n}\right\},\left\{\beta_{n}\right\}$ are two sequences in $[0,1]$ satisfying the conditions:

(i) $\alpha_{n}+\beta_{n} \leq 1$ for all $n \in N$;

(ii) $\lim _{n \rightarrow \infty} \alpha_{n}=0$;

(iii) $\liminf _{n \rightarrow \infty} \beta_{n}>0$.

Then, $\left\{x_{n}\right\},\left\{u_{n}\right\}$ and $\left\{z_{n}\right\}$ converge strongly to $w=P_{F i x(S) \cap G M E P(F, \varphi, B)}(x)$.

Proof. Putting $A=0$, by Theorem 3.1 we obtain the desired result.

Theorem 3.3. Let $C$ be a nonempty closed convex subset of a real Hilbert space $H$. Let $F$ be a bifunction from $C \times C$ to $R$ satisfying (A1)-(A5) and $\varphi: C \rightarrow R$ be a lower semicontinuous and convex function. Let $B$ be an $\alpha$-inverse-strongly monotone mapping of $C$ into $H$. Let $S$ be a nonexpansive mapping of $C$ into $H$ such that Fix $(S) \cap G M E P(F, \varphi, B) \neq \emptyset$. Assume that either (B1) or (B2) holds. Let $\left\{x_{n}\right\},\left\{u_{n}\right\}$ and $\left\{z_{n}\right\}$ be sequences generated by

$$
\left\{\begin{array}{l}
x_{1}=x \in C, \\
F\left(u_{n}, y\right)+\varphi(y)-\varphi\left(u_{n}\right)+\left\langle B x_{n}, y-u_{n}\right\rangle+\frac{1}{r_{n}}\left\langle y-u_{n}, u_{n}-x_{n}\right\rangle \geq 0, \quad \forall y \in C, \\
z_{n}=\left(1-\beta_{n}\right) x_{n}+\beta_{n} S u_{n}, \\
C_{n}=\left\{z \in C:\left\|z_{n}-z\right\| \leq\left\|x_{n}-z\right\|\right\}, \\
Q_{n}=\left\{z \in C:\left\langle x_{n}-z, x-x_{n}\right\rangle \geq 0\right\}, \\
x_{n+1}=P_{C_{n}} \cap Q_{n} x
\end{array}\right.
$$

for every $n=1,2, \ldots$ where $\left\{r_{n}\right\} \subset[d, e]$ for some $d, e \in(0,2 \alpha)$, and $\left\{\beta_{n}\right\}$ is a sequence in $[0,1]$ satisfying the condition $\liminf _{n \rightarrow \infty} \beta_{n}>0$. Then, $\left\{x_{n}\right\},\left\{u_{n}\right\}$ and $\left\{z_{n}\right\}$ converge strongly to $w=P_{F i x(S) \cap G M E P(F, \varphi, B)}(x)$.

Proof. Putting $A=0$ and $\alpha_{n}=0$ for every $n=1,2, \ldots$, by Theorem 3.1 we obtain the desired result. 
A mapping $T$ of a closed convex subset $C$ into itself is pseudocontractive if there holds that

$$
\langle T x-T y, x-y\rangle \leq\|x-y\|^{2}
$$

for all $x, y \in C$; see [33]. Obviously, the class of pseudocontractive mappings is more general than the class of nonexpansive mappings. Now we prove a strong convergence theorem of a new iterative process for finding a common element of the set of solutions of a generalized mixed equilibrium problem, the set of fixed points of a nonexpansive mapping and the set of fixed points of a Lipschitz pseudocontractive mapping.

Theorem 3.4. Let $C$ be a nonempty closed convex subset of a real Hilbert space $H$. Let $F$ be a bifunction from $C \times C$ to $R$ satisfying $(A 1)-(A 5)$ and $\varphi: C \rightarrow R$ be a lower semicontinuous and convex function. Let $T$ be a pseudocontrative and $m$-Lipschitz-continuous mapping of $C$ into itself and $B$ be an $\alpha$-inverse-strongly monotone mapping of $C$ into $H$. Let $S$ be a nonexpansive mapping of $C$ into $H$ such that $\operatorname{Fix}(S) \cap \cap F i x(T) \cap G M E P(F, \varphi, B) \neq \emptyset$. Assume that either (B1) or (B2) holds. Let $\left\{x_{n}\right\},\left\{u_{n}\right\},\left\{y_{n}\right\}$ and $\left\{z_{n}\right\}$ be sequences generated by

$$
\left\{\begin{array}{l}
x_{1}=x \in C, \\
F\left(u_{n}, y\right)+\varphi(y)-\varphi\left(u_{n}\right)+\left\langle B x_{n}, y-u_{n}\right\rangle+\frac{1}{r_{n}}\left\langle y-u_{n}, u_{n}-x_{n}\right\rangle \geq 0, \quad \forall y \in C, \\
y_{n}=\left(1-\gamma_{n}\right) u_{n}+\gamma_{n}\left[u_{n}-\lambda_{n}\left(u_{n}-T u_{n}\right)\right], \\
z_{n}=\left(1-\alpha_{n}-\beta_{n}\right) x_{n}+\alpha_{n} y_{n}+\beta_{n} S P_{C}\left(u_{n}-\lambda_{n}\left(y_{n}-T y_{n}\right)\right), \\
C_{n}=\left\{z \in C:\left\|z_{n}-z\right\|^{2} \leq\left\|x_{n}-z\right\|^{2}+\left(3-3 \gamma_{n}+\alpha_{n}\right) b^{2}\left\|u_{n}-T u_{n}\right\|^{2}\right\}, \\
Q_{n}=\left\{z \in C:\left\langle x_{n}-z, x-x_{n}\right\rangle \geq 0\right\}, \\
x_{n+1}=P_{C_{n} \cap Q_{n}} x
\end{array}\right.
$$

for every $n=1,2, \ldots$ where $\left\{\lambda_{n}\right\} \subset[a, b]$ for some $[a, b] \subset\left(0, \frac{1}{4(m+1)}\right),\left\{r_{n}\right\} \subset$ $[d, e]$ for some $d, e \in(0,2 \alpha)$, and $\left\{\alpha_{n}\right\},\left\{\beta_{n}\right\},\left\{\gamma_{n}\right\}$ are three sequences in $[0,1]$ satisfying the conditions:

(i) $\alpha_{n}+\beta_{n} \leq 1$ for all $n \in N$;

(ii) $\lim _{n \rightarrow \infty} \alpha_{n}=0$;

(iii) $\liminf _{n \rightarrow \infty} \beta_{n}>0$;

(iv) $\lim _{n \rightarrow \infty} \gamma_{n}=1$ and $\gamma_{n}>\frac{3}{4}$ for all $n \in N$;

Then, $\left\{x_{n}\right\},\left\{u_{n}\right\},\left\{y_{n}\right\}$ and $\left\{z_{n}\right\}$ converge strongly to $w=P_{\text {Fix }(S) \cap \cap F i x(T) \cap G M E P(F, \varphi, B)}(x)$.

Proof. Let $A=I-T$. From the proof of Theorem 4.5 in [27], we know that the mapping $A$ is monotone and $(m+1)$-Lipschitz-continuous and $F i x(T)=V I(C, A)$. By Theorem 3.1 we obtain the desired result. 
Theorem 3.5. Let $C$ be a nonempty closed convex subset of a real Hilbert space $H$. Let $F$ be a bifunction from $C \times C$ to $R$ satisfying $(A 1)-(A 5)$ and $\varphi: C \rightarrow R$ be a lower semicontinuous and convex function. Let $B$ be an $\alpha$-inverse-strongly monotone mapping of $C$ into $H$ and $A$ be a monotone and $k$-Lipschitz-continuous mapping of $C$ into $H$. Let $S$ be a nonexpansive mapping of $C$ into $H$ such that $\Omega=F i x(S) \cap V I(C, A) \cap G M E P(F, \varphi, B) \neq \emptyset$. Assume that either $(B 1)$ or $(B 2)$ holds. Let $\left\{x_{n}\right\},\left\{u_{n}\right\},\left\{y_{n}\right\}$ and $\left\{z_{n}\right\}$ be sequences generated by

$$
\left\{\begin{array}{l}
x_{1}=x \in C, \\
F\left(u_{n}, y\right)+\varphi(y)-\varphi\left(u_{n}\right)+\left\langle B x_{n}, y-u_{n}\right\rangle+\frac{1}{r_{n}}\left\langle y-u_{n}, u_{n}-x_{n}\right\rangle \geq 0, \quad \forall y \in C, \\
y_{n}=P_{C}\left(u_{n}-\lambda_{n} A u_{n}\right), \\
z_{n}=\left(1-\beta_{n}\right) x_{n}+\beta_{n} S P_{C}\left(u_{n}-\lambda_{n} A y_{n}\right), \\
C_{n}=\left\{z \in C:\left\|z_{n}-z\right\|^{2} \leq\left\|x_{n}-z\right\|^{2}\right\}, \\
Q_{n}=\left\{z \in C:\left\langle x_{n}-z, x-x_{n}\right\rangle \geq 0\right\}, \\
x_{n+1}=P_{C_{n} \cap Q_{n}} x
\end{array}\right.
$$

for every $n=1,2, \ldots$ where $\left\{\lambda_{n}\right\} \subset[a, b]$ for some $[a, b] \subset\left(0, \frac{1}{4 k}\right),\left\{r_{n}\right\} \subset[d, e]$ for some $d, e \in(0,2 \alpha)$, and $\left\{\beta_{n}\right\}$ is a sequence in $[0,1]$ satisfying $\liminf _{n \rightarrow \infty} \beta_{n}>0$. Then, $\left\{x_{n}\right\},\left\{u_{n}\right\},\left\{y_{n}\right\}$ and $\left\{z_{n}\right\}$ converge strongly to $w=P_{\Omega}(x)$.

Proof. Putting $\gamma_{n}=1$ and $\alpha_{n}=0$, by Theorem 3.1 we obtain the desired result.

It is easy to see that Theorem 3.1-3.5 generalize and extend Theorem 3.1 in [9].

Theorem 3.6. Let $C$ be a nonempty closed convex subset of a real Hilbert space $H$. Let $F$ be a bifunction from $C \times C$ to $R$ satisfying $(A 1)-(A 5)$ and $\varphi: C \rightarrow R$ be a lower semicontinuous and convex function. Let $B$ be an $\alpha$-inverse-strongly monotone mapping of $C$ into $H$ and $A$ be a monotone and $k$-Lipschitz-continuous mapping of $C$ into $H$. Let $S$ be a nonexpansive mapping of $C$ into $H$ such that $\Omega=\operatorname{Fix}(S) \cap V I(C, A) \cap \operatorname{GMEP}(F, \varphi, B) \neq \emptyset$. Assume that either (B1) or (B2) holds. Let $\left\{x_{n}\right\},\left\{u_{n}\right\},\left\{y_{n}\right\}$ and $\left\{z_{n}\right\}$ be sequences generated by

$$
\left\{\begin{array}{l}
x_{1}=x \in C, \\
F\left(u_{n}, y\right)+\varphi(y)-\varphi\left(u_{n}\right)+\left\langle B x_{n}, y-u_{n}\right\rangle+\frac{1}{r_{n}}\left\langle y-u_{n}, u_{n}-x_{n}\right\rangle \geq 0, \quad \forall y \in C, \\
y_{n}=P_{C}\left(u_{n}-\lambda_{n} A u_{n}\right), \\
z_{n}=P_{C}\left(u_{n}-\lambda_{n} A y_{n}\right), \\
C_{n}=\left\{z \in C:\left\|z_{n}-z\right\|^{2} \leq\left\|x_{n}-z\right\|^{2}\right\}, \\
Q_{n}=\left\{z \in C:\left\langle x_{n}-z, x-x_{n}\right\rangle \geq 0\right\}, \\
x_{n+1}=P_{C_{n}} \cap Q_{n} x
\end{array}\right.
$$


for every $n=1,2, \ldots$ where $\left\{\lambda_{n}\right\} \subset[a, b]$ for some $[a, b] \subset\left(0, \frac{1}{4 k}\right),\left\{r_{n}\right\} \subset[d, e]$ for some $d, e \in(0,2 \alpha)$. Then, $\left\{x_{n}\right\},\left\{u_{n}\right\},\left\{y_{n}\right\}$ and $\left\{z_{n}\right\}$ converge strongly to $w=P_{\Omega}(x)$.

Proof. Putting Putting $S=I, \gamma_{n}=\beta_{n}=1$ and $\alpha_{n}=0$ for every $n=1,2, \ldots$, by Theorem 3.1 we obtain the desired result.

Theorem 3.7 Let $C$ be a nonempty closed convex subset of a real Hilbert space $H$. Let $F$ be a bifunction from $C \times C$ to $R$ satisfying (A1)-(A5) and $\varphi$ : $C \rightarrow R$ be a lower semicontinuous and convex function. Let $A$ be a monotone and $k$-Lipschitz-continuous mapping of $C$ into $H$ and $B$ be an $\alpha$-inverse-strongly monotone mapping of $C$ into $H$. Let $S$ be a nonexpansive mapping of $C$ into $H$ such that $\Omega=F i x(S) \cap V I(C, A) \cap \operatorname{GMEP}(F, \varphi, B) \neq \emptyset$. Assume that either (B1) or (B2) holds. Let $\left\{x_{n}\right\},\left\{u_{n}\right\},\left\{y_{n}\right\}$ and $\left\{z_{n}\right\}$ be sequences generated by

$$
\left\{\begin{array}{l}
x_{1}=x \in C, \\
F\left(u_{n}, y\right)+\varphi(y)-\varphi\left(u_{n}\right)+\left\langle B x_{n}, y-u_{n}\right\rangle+\frac{1}{r_{n}}\left\langle y-u_{n}, u_{n}-x_{n}\right\rangle \geq 0, \quad \forall y \in C, \\
y_{n}=\left(1-\gamma_{n}\right) u_{n}+\gamma_{n} P_{C}\left(u_{n}-\lambda_{n} A u_{n}\right), \\
z_{n}=P_{C}\left(u_{n}-\lambda_{n} A y_{n}\right), \\
C_{n}=\left\{z \in C:\left\|z_{n}-z\right\|^{2} \leq\left\|x_{n}-z\right\|^{2}+\left(3-3 \gamma_{n}+\alpha_{n}\right) b^{2}\left\|A u_{n}\right\|^{2}\right\}, \\
Q_{n}=\left\{z \in C:\left\langle x_{n}-z, x-x_{n}\right\rangle \geq 0\right\}, \\
x_{n+1}=P_{C_{n} \cap Q_{n}} x
\end{array}\right.
$$

for every $n=1,2, \ldots$ where $\left\{\lambda_{n}\right\} \subset[a, b]$ for some $[a, b] \subset\left(0, \frac{1}{4 k}\right),\left\{r_{n}\right\} \subset[d, e]$ for some $d, e \in(0,2 \alpha)$, and $\left\{\gamma_{n}\right\}$ is a sequence in $[0,1]$ such that $\lim _{n \rightarrow \infty} \gamma_{n}=1$ and $\gamma_{n}>\frac{3}{4}$ for all $n \in N$. Then, $\left\{x_{n}\right\},\left\{u_{n}\right\},\left\{y_{n}\right\}$ and $\left\{z_{n}\right\}$ converge strongly to $w=P_{\Omega}(x)$.

Proof. Putting $S=I, \alpha_{n}=0$ and $\beta_{n}=1$ for every $n=1,2, \ldots$, by Theorem 3.1 we obtain the desired result.

\section{Applications}

By the above results, we can obtain many new and interesting strong convergence theorems for some algorithms of finding the solution of the problems (1.2)-(1.7). Now we give some examples as follows:

Let $B=0$, by Theorem 3.1 and 3.5, we obtain the following two strong convergence theorems for the algorithms of finding solutions of problem (1.2):

Theorem 4.1. Let $C$ be a nonempty closed convex subset of a real Hilbert space $H$. Let $F$ be a bifunction from $C \times C$ to $R$ satisfying $(A 1)-(A 5)$ and 
$\varphi: C \rightarrow R$ be a lower semicontinuous and convex function. Let $A$ be a monotone and $k$-Lipschitz-continuous mapping of $C$ into $H$. Let $S$ be a nonexpansive mapping of $C$ into $H$ such that Fix $(S) \cap V I(C, A) \cap M E P(F, \varphi) \neq \emptyset$. Assume that either (B1) or (B2) holds. Let $\left\{x_{n}\right\},\left\{u_{n}\right\},\left\{y_{n}\right\}$ and $\left\{z_{n}\right\}$ be sequences generated by

$$
\left\{\begin{array}{l}
x_{1}=x \in C, \\
F\left(u_{n}, y\right)+\varphi(y)-\varphi\left(u_{n}\right)+\frac{1}{r_{n}}\left\langle y-u_{n}, u_{n}-x_{n}\right\rangle \geq 0, \quad \forall y \in C, \\
y_{n}=\left(1-\gamma_{n}\right) u_{n}+\gamma_{n} P_{C}\left(u_{n}-\lambda_{n} A u_{n}\right), \\
z_{n}=\left(1-\alpha_{n}-\beta_{n}\right) x_{n}+\alpha_{n} y_{n}+\beta_{n} S P_{C}\left(u_{n}-\lambda_{n} A y_{n}\right), \\
C_{n}=\left\{z \in C:\left\|z_{n}-z\right\|^{2} \leq\left\|x_{n}-z\right\|^{2}+\left(3-3 \gamma_{n}+\alpha_{n}\right) b^{2}\left\|A u_{n}\right\|^{2}\right\}, \\
Q_{n}=\left\{z \in C:\left\langle x_{n}-z, x-x_{n}\right\rangle \geq 0\right\}, \\
x_{n+1}=P_{C_{n} \cap Q_{n}} x
\end{array}\right.
$$

for every $n=1,2, \ldots$ where $\left\{\lambda_{n}\right\} \subset[a, b]$ for some $[a, b] \subset\left(0, \frac{1}{4 k}\right),\left\{r_{n}\right\} \subset$ $[d,+\infty)$ for some $d>0$, and $\left\{\alpha_{n}\right\},\left\{\beta_{n}\right\},\left\{\gamma_{n}\right\}$ are three sequences in $[0,1]$ satisfying the conditions:

(i) $\alpha_{n}+\beta_{n} \leq 1$ for all $n \in N$;

(ii) $\lim _{n \rightarrow \infty} \alpha_{n}=0$;

(iii) $\liminf _{n \rightarrow \infty} \beta_{n}>0$;

(iv) $\lim _{n \rightarrow \infty} \gamma_{n}=1$ and $\gamma_{n}>\frac{3}{4}$ for all $n \in N$;

Then, $\left\{x_{n}\right\},\left\{u_{n}\right\},\left\{y_{n}\right\}$ and $\left\{z_{n}\right\}$ converge strongly to $w=P_{F i x(S) \cap V I(C, A) \cap M E P(F, \varphi)}(x)$.

Theorem 4.2. Let $C$ be a nonempty closed convex subset of a real Hilbert space $H$. Let $F$ be a bifunction from $C \times C$ to $R$ satisfying $(A 1)-(A 5)$ and $\varphi: C \rightarrow R$ be a lower semicontinuous and convex function. Let $A$ be a monotone and $k$-Lipschitz-continuous mapping of $C$ into $H$. Let $S$ be a nonexpansive mapping of $C$ into $H$ such that Fix $(S) \cap V I(C, A) \cap M E P(F, \varphi) \neq \emptyset$. Assume that either (B1) or (B2) holds. Let $\left\{x_{n}\right\},\left\{u_{n}\right\},\left\{y_{n}\right\}$ and $\left\{z_{n}\right\}$ be sequences generated by

$$
\left\{\begin{array}{l}
x_{1}=x \in C, \\
F\left(u_{n}, y\right)+\varphi(y)-\varphi\left(u_{n}\right)+\frac{1}{r_{n}}\left\langle y-u_{n}, u_{n}-x_{n}\right\rangle \geq 0, \quad \forall y \in C, \\
y_{n}=P_{C}\left(u_{n}-\lambda_{n} A u_{n}\right), \\
z_{n}=\left(1-\beta_{n}\right) x_{n}+\beta_{n} S P_{C}\left(u_{n}-\lambda_{n} A y_{n}\right), \\
C_{n}=\left\{z \in C:\left\|z_{n}-z\right\|^{2} \leq\left\|x_{n}-z\right\|^{2}\right\} \\
Q_{n}=\left\{z \in C:\left\langle x_{n}-z, x-x_{n}\right\rangle \geq 0\right\} \\
x_{n+1}=P_{C_{n} \cap Q_{n}} x
\end{array}\right.
$$


for every $n=1,2, \ldots$ where $\left\{\lambda_{n}\right\} \subset[a, b]$ for some $[a, b] \subset\left(0, \frac{1}{4 k}\right),\left\{r_{n}\right\} \subset$ $[d,+\infty)$ for some $d>0$, and $\left\{\beta_{n}\right\}$ is a sequence in $[0,1]$ satisfying $\liminf _{n \rightarrow \infty} \beta_{n}>0$.

Then, $\left\{x_{n}\right\},\left\{u_{n}\right\},\left\{y_{n}\right\}$ and $\left\{z_{n}\right\}$ converge strongly to

$w=P_{F i x(S) \cap V I(C, A) \cap M E P(F, \varphi)}(x)$.

Let $\varphi=0$, by Theorem 3.1 and 3.5, we obtain the following two strong convergence theorems for the algorithms of finding solutions of problem (1.3):

Theorem 4.3. Let $C$ be a nonempty closed convex subset of a real Hilbert space $H$. Let $F$ be a bifunction from $C \times C$ to $R$ satisfying $(A 1)-(A 5)$. Let $A$ be a monotone and k-Lipschitz-continuous mapping of $C$ into $H$ and $B$ be an $\alpha$ inverse-strongly monotone mapping of $C$ into $H$. Let $S$ be a nonexpansive mapping of $C$ into $H$ such that Fix $(S) \cap V I(C, A) \cap G E P(F, B) \neq \emptyset$. Assume that either (B4) or (B2) holds. Let $\left\{x_{n}\right\},\left\{u_{n}\right\},\left\{y_{n}\right\}$ and $\left\{z_{n}\right\}$ be sequences generated by

$$
\left\{\begin{array}{l}
x_{1}=x \in C, \\
F\left(u_{n}, y\right)+\left\langle B x_{n}, y-u_{n}\right\rangle+\frac{1}{r_{n}}\left\langle y-u_{n}, u_{n}-x_{n}\right\rangle \geq 0, \quad \forall y \in C, \\
y_{n}=\left(1-\gamma_{n}\right) u_{n}+\gamma_{n} P_{C}\left(u_{n}-\lambda_{n} A u_{n}\right), \\
z_{n}=\left(1-\alpha_{n}-\beta_{n}\right) x_{n}+\alpha_{n} y_{n}+\beta_{n} S P_{C}\left(u_{n}-\lambda_{n} A y_{n}\right), \\
C_{n}=\left\{z \in C:\left\|z_{n}-z\right\|^{2} \leq\left\|x_{n}-z\right\|^{2}+\left(3-3 \gamma_{n}+\alpha_{n}\right) b^{2}\left\|A u_{n}\right\|^{2}\right\}, \\
Q_{n}=\left\{z \in C:\left\langle x_{n}-z, x-x_{n}\right\rangle \geq 0\right\}, \\
x_{n+1}=P_{C_{n}} \cap Q_{n} x
\end{array}\right.
$$

for every $n=1,2, \ldots$ where $\left\{\lambda_{n}\right\} \subset[a, b]$ for some $[a, b] \subset\left(0, \frac{1}{4 k}\right),\left\{r_{n}\right\} \subset$ $[d, e]$ for some $d, e \in(0,2 \alpha)$, and $\left\{\alpha_{n}\right\},\left\{\beta_{n}\right\},\left\{\gamma_{n}\right\}$ are three sequences in $[0,1]$ satisfying the conditions:

(i) $\alpha_{n}+\beta_{n} \leq 1$ for all $n \in N$;

(ii) $\lim _{n \rightarrow \infty} \alpha_{n}=0$;

(iii) $\liminf _{n \rightarrow \infty} \beta_{n}>0$;

(iii) $\lim _{n \rightarrow \infty} \gamma_{n}=1$ and $\gamma_{n}>\frac{3}{4}$ for all $n \in N$;

Then, $\left\{x_{n}\right\},\left\{u_{n}\right\},\left\{y_{n}\right\}$ and $\left\{z_{n}\right\}$ converge strongly to $w=P_{F i x(S) \cap V I(C, A) \cap G E P(F, B)}(x)$.

Theorem 4.4. Let $C$ be a nonempty closed convex subset of a real Hilbert space $H$. Let $F$ be a bifunction from $C \times C$ to $R$ satisfying $(A 1)-(A 5)$. Let $A$ be a monotone and k-Lipschitz-continuous mapping of $C$ into $H$ and $B$ be an $\alpha$ inverse-strongly monotone mapping of $C$ into $H$. Let $S$ be a nonexpansive mapping 
of $C$ into $H$ such that Fix $(S) \cap V I(C, A) \cap G E P(F, B) \neq \emptyset$. Assume that either (B4) or (B2) holds. Let $\left\{x_{n}\right\},\left\{u_{n}\right\},\left\{y_{n}\right\}$ and $\left\{z_{n}\right\}$ be sequences generated by

$$
\left\{\begin{array}{l}
x_{1}=x \in C, \\
F\left(u_{n}, y\right)+\left\langle B x_{n}, y-u_{n}\right\rangle+\frac{1}{r_{n}}\left\langle y-u_{n}, u_{n}-x_{n}\right\rangle \geq 0, \quad \forall y \in C, \\
y_{n}=P_{C}\left(u_{n}-\lambda_{n} A u_{n}\right), \\
z_{n}=\left(1-\beta_{n}\right) x_{n}+\beta_{n} S P_{C}\left(u_{n}-\lambda_{n} A y_{n}\right), \\
C_{n}=\left\{z \in C:\left\|z_{n}-z\right\|^{2} \leq\left\|x_{n}-z\right\|^{2}\right\}, \\
Q_{n}=\left\{z \in C:\left\langle x_{n}-z, x-x_{n}\right\rangle \geq 0\right\}, \\
x_{n+1}=P_{C_{n}} \cap Q_{n} x
\end{array}\right.
$$

for every $n=1,2, \ldots$ where $\left\{\lambda_{n}\right\} \subset[a, b]$ for some $[a, b] \subset\left(0, \frac{1}{4 k}\right),\left\{r_{n}\right\} \subset[d, e]$ for some $d, e \in(0,2 \alpha)$, and $\left\{\beta_{n}\right\}$ is a sequence in $[0,1]$ satisfying $\liminf _{n \rightarrow \infty} \beta_{n}>0$.

Then, $\left\{x_{n}\right\},\left\{u_{n}\right\},\left\{y_{n}\right\}$ and $\left\{z_{n}\right\}$ converge strongly to $w=P_{F i x(S) \cap V I(C, A) \cap G E P(F, B)}(x)$.

Let $F(x, y)=0$ for $x, y \in C$, by Theorem 3.1 we obtain the following strong convergence theorem for an algorithm of finding solutions of problem (1.5):

Theorem 4.5. Let $C$ be a nonempty closed convex subset of a real Hilbert space $H$. Let $\varphi: C \rightarrow R$ be a lower semicontinuous and convex function. Let $A$ be a monotone and k-Lipschitz-continuous mapping of $C$ into $H$ and $B$ be an $\alpha$ inverse-strongly monotone mapping of $C$ into $H$. Let $S$ be a nonexpansive mapping of $C$ into $H$ such that Fix $(S) \cap V I(C, A) \cap G V I(C, \varphi, B) \neq \emptyset$. Assume that either (B3) or (B2) holds. Let $\left\{x_{n}\right\},\left\{u_{n}\right\},\left\{y_{n}\right\}$ and $\left\{z_{n}\right\}$ be sequences generated by

$$
\left\{\begin{array}{l}
x_{1}=x \in C, \\
\varphi(y)-\varphi\left(u_{n}\right)+\left\langle B x_{n}, y-u_{n}\right\rangle+\frac{1}{r_{n}}\left\langle y-u_{n}, u_{n}-x_{n}\right\rangle \geq 0, \quad \forall y \in C, \\
y_{n}=\left(1-\gamma_{n}\right) u_{n}+\gamma_{n} P_{C}\left(u_{n}-\lambda_{n} A u_{n}\right) \\
z_{n}=\left(1-\alpha_{n}-\beta_{n}\right) x_{n}+\alpha_{n} y_{n}+\beta_{n} S P_{C}\left(u_{n}-\lambda_{n} A y_{n}\right), \\
C_{n}=\left\{z \in C:\left\|z_{n}-z\right\|^{2} \leq\left\|x_{n}-z\right\|^{2}+\left(3-3 \gamma_{n}+\alpha_{n}\right) b^{2}\left\|A u_{n}\right\|^{2}\right\} \\
Q_{n}=\left\{z \in C:\left\langle x_{n}-z, x-x_{n}\right\rangle \geq 0\right\} \\
x_{n+1}=P_{C_{n}} \cap Q_{n} x
\end{array}\right.
$$

for every $n=1,2, \ldots$ where $\left\{\lambda_{n}\right\} \subset[a, b]$ for some $[a, b] \subset\left(0, \frac{1}{4 k}\right),\left\{r_{n}\right\} \subset$ $[d, e]$ for some $d, e \in(0,2 \alpha)$, and $\left\{\alpha_{n}\right\},\left\{\beta_{n}\right\},\left\{\gamma_{n}\right\}$ are three sequences in $[0,1]$ satisfying the conditions:

(i) $\alpha_{n}+\beta_{n} \leq 1$ for all $n \in N$; 
(ii) $\lim _{n \rightarrow \infty} \alpha_{n}=0$;

(iii) $\liminf _{n \rightarrow \infty} \beta_{n}>0$;

(iv) $\lim _{n \rightarrow \infty} \gamma_{n}=1$ and $\gamma_{n}>\frac{3}{4}$ for all $n \in N$;

Then, $\left\{x_{n}\right\},\left\{u_{n}\right\},\left\{y_{n}\right\}$ and $\left\{z_{n}\right\}$ converge strongly to $w=P_{F i x(S) \cap V I(C, A) \cap G V I(C, \varphi, B)}(x)$.

Let $B=0$ and $F(x, y)=0$ for $x, y \in C$, by Theorem 3.1 we obtain the following strong convergence theorem for an algorithm of finding solutions of problem (1.7):

Theorem 4.6. Let $C$ be a nonempty closed convex subset of a real Hilbert space $H$. Let $\varphi: C \rightarrow R$ be a lower semicontinuous and convex function. Let $A$ be a monotone and k-Lipschitz-continuous mapping of $C$ into $H$. Let $S$ be a nonexpansive mapping of $C$ into $H$ such that $F i x(S) \cap V I(C, A) \cap \operatorname{Argmin}(\varphi) \neq \emptyset$. Assume that either (B3) or (B2) holds. Let $\left\{x_{n}\right\},\left\{u_{n}\right\},\left\{y_{n}\right\}$ and $\left\{z_{n}\right\}$ be sequences generated by

$$
\left\{\begin{array}{l}
x_{1}=x \in C, \\
\varphi(y)-\varphi\left(u_{n}\right)+\frac{1}{r_{n}}\left\langle y-u_{n}, u_{n}-x_{n}\right\rangle \geq 0, \quad \forall y \in C, \\
y_{n}=\left(1-\gamma_{n}\right) u_{n}+\gamma_{n} P_{C}\left(u_{n}-\lambda_{n} A u_{n}\right), \\
z_{n}=\left(1-\alpha_{n}-\beta_{n}\right) x_{n}+\alpha_{n} y_{n}+\beta_{n} S P_{C}\left(u_{n}-\lambda_{n} A y_{n}\right), \\
C_{n}=\left\{z \in C:\left\|z_{n}-z\right\|^{2} \leq\left\|x_{n}-z\right\|^{2}+\left(3-3 \gamma_{n}+\alpha_{n}\right) b^{2}\left\|A u_{n}\right\|^{2}\right\} \\
Q_{n}=\left\{z \in C:\left\langle x_{n}-z, x-x_{n}\right\rangle \geq 0\right\} \\
x_{n+1}=P_{C_{n}} \cap Q_{n} x
\end{array}\right.
$$

for every $n=1,2, \ldots$ where $\left\{\lambda_{n}\right\} \subset[a, b]$ for some $[a, b] \subset\left(0, \frac{1}{4 k}\right),\left\{r_{n}\right\} \subset$ $[d,+\infty)$ for some $d>0$, and $\left\{\alpha_{n}\right\},\left\{\beta_{n}\right\},\left\{\gamma_{n}\right\}$ are three sequences in $[0,1]$ satisfying the conditions:

(i) $\alpha_{n}+\beta_{n} \leq 1$ for all $n \in N$;

(ii) $\lim _{n \rightarrow \infty} \alpha_{n}=0$;

(iii) $\liminf _{n \rightarrow \infty} \beta_{n}>0$;

(iv) $\lim _{n \rightarrow \infty} \gamma_{n}=1$ and $\gamma_{n}>\frac{3}{4}$ for all $n \in N$;

Then, $\left\{x_{n}\right\},\left\{u_{n}\right\},\left\{y_{n}\right\}$ and $\left\{z_{n}\right\}$ converge strongly to $w=P_{F i x(S) \cap V I(C, A) \cap \operatorname{Argmin}(\varphi)}(x)$.

Let $B=0$ and $\varphi=0$, by Theorem 3.1, we obtain the following strong convergence theorem for an algorithm of finding solutions of problem (1.4): 
Theorem 4.7. Let $C$ be a nonempty closed convex subset of a real Hilbert space $H$. Let $F$ be a bifunction from $C \times C$ to $R$ satisfying $(A 1)-(A 5)$. Let $A$ be a monotone and $k$-Lipschitz-continuous mapping of $C$ into $H$. Let $S$ be a nonexpansive mapping of $C$ into $H$ such that $F i x(S) \cap V I(C, A) \cap M E P(F, \varphi) \neq$ $\emptyset$. Assume that either $(B 4)$ or (B2) holds. Let $\left\{x_{n}\right\},\left\{u_{n}\right\},\left\{y_{n}\right\}$ and $\left\{z_{n}\right\}$ be sequences generated by

$$
\left\{\begin{array}{l}
x_{1}=x \in C, \\
F\left(u_{n}, y\right)+\frac{1}{r_{n}}\left\langle y-u_{n}, u_{n}-x_{n}\right\rangle \geq 0, \quad \forall y \in C, \\
y_{n}=\left(1-\gamma_{n}\right) u_{n}+\gamma_{n} P_{C}\left(u_{n}-\lambda_{n} A u_{n}\right), \\
z_{n}=\left(1-\alpha_{n}-\beta_{n}\right) x_{n}+\alpha_{n} y_{n}+\beta_{n} S P_{C}\left(u_{n}-\lambda_{n} A y_{n}\right), \\
C_{n}=\left\{z \in C:\left\|z_{n}-z\right\|^{2} \leq\left\|x_{n}-z\right\|^{2}+\left(3-3 \gamma_{n}+\alpha_{n}\right) b^{2}\left\|A u_{n}\right\|^{2}\right\} \\
Q_{n}=\left\{z \in C:\left\langle x_{n}-z, x-x_{n}\right\rangle \geq 0\right\} \\
x_{n+1}=P_{C_{n} \cap Q_{n}} x
\end{array}\right.
$$

for every $n=1,2, \ldots$ where $\left\{\lambda_{n}\right\} \subset[a, b]$ for some $[a, b] \subset\left(0, \frac{1}{4 k}\right),\left\{r_{n}\right\} \subset$ $[d,+\infty)$ for some $d>0$, and $\left\{\alpha_{n}\right\},\left\{\beta_{n}\right\},\left\{\gamma_{n}\right\}$ are three sequences in $[0,1]$ satisfying the conditions:

(i) $\alpha_{n}+\beta_{n} \leq 1$ for all $n \in N$;

(ii) $\lim _{n \rightarrow \infty} \alpha_{n}=0$;

(iii) $\liminf _{n \rightarrow \infty} \beta_{n}>0$;

(iv) $\lim _{n \rightarrow \infty} \gamma_{n}=1$ and $\gamma_{n}>\frac{3}{4}$ for all $n \in N$;

Then, $\left\{x_{n}\right\},\left\{u_{n}\right\},\left\{y_{n}\right\}$ and $\left\{z_{n}\right\}$ converge strongly to $w=P_{F i x(S) \cap V I(C, A) \cap E P(F)}(x)$.

Theorem 4.8. (see Theorem 5 in [28]). Let $C$ be a nonempty closed convex subset of a real Hilbert space $H$. Let $A$ be a monotone and $k$-Lipschitz-continuous mapping of $C$ into $H$. Let $S$ be a nonexpansive mapping of $C$ into $H$ such that Fix $(S) \cap V I(C, A) \neq \emptyset$. Let $\left\{x_{n}\right\},\left\{y_{n}\right\}$ and $\left\{z_{n}\right\}$ be sequences generated by (1.12), where $\left\{\lambda_{n}\right\} \subset[a, b]$ for some $[a, b] \subset\left(0, \frac{1}{4 k}\right)$ and $\left\{\alpha_{n}\right\},\left\{\beta_{n}\right\},\left\{\gamma_{n}\right\}$ are three sequences in $[0,1]$ satisfying the conditions:

(i) $\alpha_{n}+\beta_{n} \leq 1$ for all $n \in N$;

(ii) $\lim _{n \rightarrow \infty} \alpha_{n}=0$;

(iii) $\liminf _{n \rightarrow \infty} \beta_{n}>0$;

(iv) $\lim _{n \rightarrow \infty} \gamma_{n}=1$ and $\gamma_{n}>\frac{3}{4}$ for all $n \in N$; 
Then, $\left\{x_{n}\right\},\left\{y_{n}\right\}$ and $\left\{z_{n}\right\}$ converge strongly to $w=P_{F i x(S) \cap V I(C, A)}(x)$.

Proof. Putting $\varphi=0, B=0$ and $F(x, y)=0$ for $x, y \in C$ in Theorem 3.1 , then $u_{n}=P_{C} x_{n}=x_{n}$ for every $n=1,2, \ldots$ By Theorem 3.1 we obtain the desired result.

Theorem 4.9. (see Theorem 3.1 in [27]). Let $C$ be a nonempty closed convex subset of a real Hilbert space $H$. Let $A$ be a monotone and $k$-Lipschitz-continuous mapping of $C$ into $H$. Let $S$ be a nonexpansive mapping of $C$ into $H$ such that Fix $(S) \cap V I(C, A) \neq \emptyset$. Let $\left\{x_{n}\right\},\left\{y_{n}\right\}$ and $\left\{z_{n}\right\}$ be sequences generated by (1.11), where $\left\{\lambda_{n}\right\} \subset[a, b]$ for some $[a, b] \subset\left(0, \frac{1}{4 k}\right)$ and $\left\{\beta_{n}\right\}$ is a sequence in $[0,1]$ satisfying $\liminf _{n \rightarrow \infty} \beta_{n}>0$. Then, $\left\{x_{n}\right\},\left\{y_{n}\right\}$ and $\left\{z_{n}\right\}$ converge strongly to $w=P_{F i x(S) \cap V I(C, A)}(x)$.

Proof. Putting $\varphi=0, B=0$ and $F(x, y)=0$ for $x, y \in C, \alpha_{n}=0$ and $\gamma_{n}=1$ for every $n=1,2, \ldots$ in Theorem 3.1 , then $u_{n}=P_{C} x_{n}=x_{n}$ for every $n=1,2, \ldots$. By Theorem 3.1 we obtain the desired result.

Theorem 4.8 and 4.9 generalize and extend Theorem 3.4 in [14].

\section{REFERENCES}

1. L.-C. Ceng and J.-C. Yao, A hybrid iterative scheme for mixed equilibrium problems and fixed point problems, Journal of Computational and Applied Mathematics, 214 (2008), 186-201.

2. S. Takahashi and W. Takahashi, Strong convergence theorem for a generalized equilibrium problem and a nonexpansive mapping in a Hilbert space, Nonlinear Analysis, (2008), doi:10.10.1016/j.na.2008.02.042

3. S. D. Flam and A. S. Antipin, Equilibrium programming using proximal-like algorithms, Math. Program, 78 (1997), 29-41.

4. E. Blum and W. Oettli, From optimization and variational inequalities to equilibrium problems, Math. Stud., 63 (1994), 123-145.

5. K. Goebel and W. A. Kirk, Topics on Metric Fixed-Point Theory, Cambridge University Press, Cambridge, England, 1990.

6. P. L. Combettes and S. A. Hirstoaga, Equilibrium programming in Hilbert spaces, $J$. Nonlinear Convex Anal., 6 (2005), 117-136.

7. S. Takahashi and W. Takahashi, Viscosity approximation methods for equilibrium problems and fixed point problems in Hilbert spaces, J. Math. Anal. Appl., 331 (2006), 506-515.

8. Y. Su, M. Shang and X. Qin, An iterative method of solutions for equilibrium and optimization problems, Nonlinear Analysis (2007), doi:10.1016/j.na.2007.08.045. 
9. A. Tada and W. Takahashi, Weak and Strong Convergence Theorems for a Nonexpansive Mapping and an Equilibrium Problem, J. Optim. Theory Appl., 133 (2007), 359-370.

10. H. H. Bauschke and P. L. Combettes, A weak-to-strong convergence principle for Fej<äermonotone methods in Hilbert spaces, Math. Oper. Res., 26 (2001), 248-264.

12. H. H. Bauschke and P. L. Combettes, Construction of best Bregman approximations in reflexive Banach spaces, Proc. Amer. Math. Soc., 131 (2003), 3757-3766.

12. R. S. Burachik, J. O. Lopes, and B. F. Svaiter, An outer approximation method for the variational inequality problem, SIAM J. Control Optim., 43 (2005), 2071-2088.

13. P. L. Combettes, Strong convergence of block-iterative outer approximation methods for convex optimization, SIAM J. Control Optim., 38 (2000), 538-565.

14. K. Nakajo and W. Takahashi, Strong convergence theorems for nonexpansive mappings and nonexpansive semigroups, J. Math. Anal. Appl., 279 (2003), 372-379.

15. M. V. Solodov and B. F. Svaiter, Forcing strong convergence of proximal point iterations in a Hilbert space, Math. Program., 87 (2000), 189-202.

16. M. Kikkawa and W. Takahashi, Approximating Fixed Points of Infinite Nonexpansive Mappings by the Hybrid Method, J. Optim. Theory Appl., 117 (2003), 93-101.

17. H. Iiduka and W. Takahashi, Strong convergence theorem by a hybrid method for nonlinear mappings of nonexpansive and monotone type and applications, Adv. Nonlinear Var. Inequal., 9 (2006), 1-10.

18. G. M. Korpelevich, The extragradient method for finding saddle points and other problems, Matecon, 12 (1976), 747-756.

19. B.-S. He, Z.-H. Yang and X.-M. Yuan, An approximate proximal-extragradient type method for monotone variational inequalities, J. Math. Anal. Appl., 300 (2004), 362374.

20. R. Gárciga Otero and A. Iuzem, Proximal methods with penalization effects in Banach spaces, Numer. Funct. Anal. Optim., 25 (2004), 69-91.

21. M. V. Solodov and B. F. Svaiter, An inexact hybrid generalized proximal point algorithm and some new results on the theory of Bregman functions, Math. Oper. Res.,25 (2000), 214-230.

22. M. V. Solodov, Convergence rate analysis of iteractive algorithms for solving variational inequality problem, Math. Program., 96 (2003), 513-528.

23. L. C. Zeng and J. C. Yao, Strong convergence theorem by an extragradient method for fixed point problems and variational inequality problems, Taiwan. J. Math., 10 (2006), 1293-1303.

24. N. Nadezhkina and W. Takahashi, Weak convergence theorem by an extragradient method for nonexpansive mappings and monotone mappings, J. Optim. Theory Appl., 128 (2006), 191-201. 
25. Y. Yao and J.-C. Yao, On modified iterative method for nonexpansive mappings and monotone mappings, Appl. Math. Comput., 186(2) (2007), 1551-1558.

26. S. Plubtieng and R. Punpaeng, A new iterative method for equilibrium problems and fixed point problems of nonexpansive mappings and monotone mappings, Applied Mathematics and Computation, 197 (2008), 548-558.

27. N. Nadezhkina and W. Takahashi, Strong Convergence Theorem by a Hybrid Method for Nonexpansive Mappings and Lipschitz-continuous monotone mappings, SIAM J. Optim., 16(4) (2006), 1230-1241.

28. L. C. Ceng, N. Hadjisavvas and J. C. Yao, Strong Convergence Theorem by a Hybrid Extragradient-like Approximation Method for Variational Inequalities and Fixed point Problems, Submitted.

29. Z. Opial, Weak convergence of the sequence of successive approximation for nonexpansive mappings, Bull. Amer. Math. Soc., 73 (1967), 561-597.

30. R. T. Rockafellar, On the maximality of sums of nonlinear monotone operators, Trans. Amer. Math. Soc., 149 (1970), 75-88.

31. K. Fan, A generalization of Tychonoff's fixed-point theorem, Math. Ann., 142 (1961), 305-310.

32. K. Goebel and S. Reich, Uniform convexity, Hyperbolic Geometry, and Nonexpansive Mappings, Marcel Dekker, Inc., 1984.

33. F. E. Browder and W. V. Petryshyn, Construction of fixed points of nonlinear mappings in Hilbert space, J. Math. Anal. Appl., 20 (1967), 197-228.

Jian-Wen Peng

College of Mathematics and Computer Science,

Chongqing Normal University,

Chongqing 400047,

P. R. China

Jen-Chih Yao

Department of Applied Mathematics,

National Sun Yat-sen University,

Kaohsiung, Taiwan 804, R.O.C.

E-mail: yaojc@math.nsysu.edu.tw 